\title{
The overlapping open clusters NGC 1750 and NGC 1758
}

\author{
II. $B V R$ photographic photometry and proper motions ${ }^{\star}$ \\ D. Galadí-Enríquez ${ }^{1}$, C. Jordi ${ }^{1}$, E. Trullols ${ }^{1,2}$, J. Guibert ${ }^{3}$, K.P. Tian ${ }^{4}$, and J.L. Zhao ${ }^{4}$ \\ 1 Dept. d'Astronomia i Meteorologia, Univ. de Barcelona, Avda. Diagonal 647, E-08028 Barcelona, Spain \\ 2 Dept. de Matemàtica Aplicada i Telemàtica, Univ. Politècnica de Catalunya, Avda. Víctor Balaguer s/n, E-08800 Vilanova i \\ la Geltrú, Spain \\ 3 Centre d'Analyse des Images, Observatoire de Paris, Bâtiment Perrault, 77 Av. Denfert-Rochereau, F-75014 Paris, France \\ 4 Shanghai Astronomical Observatory, CAS, Shanghai 200030, PR China
}

Received January 28; accepted March 2, 1998

\begin{abstract}
Astrometry (positions and proper motions) and photographic $B V R$ photometry were determined from a set of 29 photographic plates and CCD data in a region of $2.3^{\circ} \times 2.3^{\circ}$ in the area of open clusters NGC 1750 and NGC 1758 in Taurus. The iterative central overlap algorithm was used for the proper motion calculations. Plateto-plate transformations and astrometric magnitude effects are discussed in detail. CCD data interact with photographic material in three different ways: for the elimination of astrometric magnitude effects, for the photometric standard calibration of photographic plates and for building a CCD pseudo-plate used for astrometric purposes as any other photographic plate. A preliminary analysis of the resulting photometric and astrometric catalogue confirmed the reality of two different clusters in the zone (NGC 1750 and NGC 1758). There were no indications for the existence of NGC 1746 .
\end{abstract}

Key words: open clusters and associations: individual: NGC 1746 - open clusters and associations: individual: NGC 1750 - open clusters and associations: individual: NGC 1758 - methods: astrometry

\section{Introduction}

Dreyer (1888), in his New General Catalogue of Nebulae and Clusters of Stars, quoted three partially overlapping clusters in a radius of $\sim 20^{\prime}$ around $\alpha_{2000}=5^{\mathrm{h}} 4^{\mathrm{m}}, \delta_{2000}=$

Send offprint requests to: D. Galadí-Enríquez

e-mail: dgaladi@pchpc5.am.ub.es

* Tables 6 and 11 are only available in electronic form from CDS via anonymous ftp to cdsarc.u-strasbg.fr (130.79.128.5) or via http://cdsweb.u-strasbg.fr/Abstract.html $+23^{\circ} 45^{\prime}:$ NGC 1746 , NGC 1750 and NGC 1758. The IAU official designations for these objects are C $0500+237$, C $0500+235$ and $\mathrm{C} 0501+237$, respectively. Their position is very close to the galactic anticenter $\left(l=179^{\circ}, b=\right.$ $\left.-11^{\circ}\right)$, in the direction of the Taurus dark cloud complex. The existence and nature of these clusters has not been definitively clarified, due to the lack of specific studies. The main analyses of this zone up to now have been the photographic photometric survey by Cuffey (1937) and the Vilnius photoelectric study by Straižys et al. (1992).

This paper is the second of a series of three devoted to a comprehensive astrometric and photometric analysis of this zone, with the aim of determining the existence of these objects and clarifying whether the possible clusters constitute a bounded, multiple system. A previous article (Galadí-Enríquez et al. 1998, from now on referred to as Paper I) was mainly devoted to the description of a $U B V R I-C C D$ photometric survey in a radius of about $15^{\prime}$ around the center of NGC 1750. Paper I contains also a brief introduction to the observational history of the area, and we refer to it for the search of literature about the clusters. The photometric data from Paper I indicated that NGC 1746 probably does not exist, and showed the presence of at least one real open cluster in the zone. This object is coincident with the position and description assigned by Dreyer (1888) and other authors to NGC 1750 and NGC 1758. It was not possible to decide the single or double nature of the cluster relying only on the positional and photometric information from Paper I.

In this article we provide additional observational material: photographic photometry, positions and proper motions in an area of $2.3^{\circ} \times 2.3^{\circ}$ around the cluster centers. The joint analysis of the CCD (Paper I) and photographic data (this paper) will lead to the clusters-field segregation and the conclusions about NGC 1746, NGC 1750 and NGC 1758 discussed in the third article of this series. 
Table 1. Plate material. The total number of plates is 30 . The maximum time difference is 103 years. The difference in epoch among deep plates is up to 39 years

\begin{tabular}{|c|c|c|c|c|c|c|c|}
\hline plate & instrument & epoch & scale $\left({ }^{\prime \prime} \mathrm{mm}^{-1}\right)$ & band & lim. mag. & use & notes \\
\hline A 550 & Calar Alto, CAHA Schmidt & 1993.88 & 85.90 & $B$ & $B \approx 20$ & astrEphot & $\mathrm{a}$ \\
\hline A 573 & Calar Alto, CAHA Schmidt & 1994.99 & 85.90 & $R$ & $R \approx 18$ & astr & $\mathrm{a}$ \\
\hline A 575 & Calar Alto, CAHA Schmidt & 1994.99 & 85.90 & $V$ & $V \approx 19$ & phot & $\mathrm{a}$ \\
\hline OCA 3305 & CERGA, OCA Schmidt & 1994.92 & 65.25 & $R$ & $R \approx 19$ & astrEphot & $\mathrm{a}, \mathrm{b}$ \\
\hline OCA 3308 & CERGA, OCA Schmidt & 1994.92 & 65.25 & $V$ & $V \approx 18$ & astr & $\mathrm{a}$ \\
\hline OCA 3314 & CERGA, OCA Schmidt & 1994.94 & 65.25 & $R$ & $R \approx 19$ & phot & $\mathrm{a}, \mathrm{c}$ \\
\hline POSS 1461-O & Mount Palomar Schmidt & 1955.82 & 67.13 & $O \sim B$ & $B \approx 20$ & astr & $\mathrm{a}, \mathrm{d}$ \\
\hline POSS 1461-E & Mount Palomar Schmidt & 1955.82 & 67.13 & $E \sim R$ & $R \approx 19$ & astr & $\mathrm{a}, \mathrm{d}$ \\
\hline T 109 & Tautenburg Schmidt & 1961.13 & 51.40 & no filter & $B \approx 19$ & astr & $\mathrm{a}$ \\
\hline T 111 & Tautenburg Schmidt & 1961.13 & 51.40 & no filter & $B \approx 18$ & astr & a \\
\hline T 6573 & Tautenburg Schmidt & 1987.98 & 51.40 & no filter & $B \approx 18$ & astr & $\mathrm{a}$ \\
\hline Т 6574 & Tautenburg Schmidt & 1987.98 & 51.40 & no filter & $B \approx 18$ & astr & $\mathrm{a}$ \\
\hline B 1118 & Heidelberg, Bruce Astrograph & 1904.94 & 103.10 & no filter & $B \approx 17$ & astr & $\mathrm{a}$ \\
\hline B 4191 & Heidelberg, Bruce Astrograph & 1919.01 & 103.10 & no filter & $B \approx 16$ & astr & $\mathrm{a}$ \\
\hline AC 2238 & Paris, AC Astrograph & 1895.09 & 60.00 & no filter & $B \approx 14$ & astr & \\
\hline AC 2239 & Paris, AC Astrograph & 1893.03 & 60.00 & no filter & $B \approx 14$ & astr & \\
\hline AC 2338 & Paris, AC Astrograph & 1892.98 & 60.00 & no filter & $B \approx 14$ & astr & \\
\hline AC 2438 & Paris, AC Astrograph & 1891.96 & 60.00 & no filter & $B \approx 14$ & astr & \\
\hline AC 2439 & Paris, AC Astrograph & 1894.06 & 60.00 & no filter & $B \approx 14$ & astr & \\
\hline S 470 & Shanghai, Zǒ-Sè Astrograph & 1918.05 & 29.89 & no filter & $B \approx 15$ & astr & \\
\hline S 471 & Shanghai, Zǒ-Sè Astrograph & 1918.08 & 29.89 & no filter & $B \approx 15$ & astr & \\
\hline S 526 & Shanghai, Zǒ-Sè Astrograph & 1930.06 & 29.89 & no filter & $B \approx 15$ & astr & \\
\hline S 527 & Shanghai, Zǒ-Sè Astrograph & 1930.08 & 29.89 & no filter & $B \approx 15$ & astr & \\
\hline S 60001 & Shanghai, Zǒ-Sè Astrograph & 1960.07 & 29.89 & no filter & $B \approx 14$ & astr & \\
\hline S 60002 & Shanghai, Zǒ-Sè Astrograph & 1960.07 & 29.89 & no filter & $B \approx 14$ & astr & \\
\hline S 83001 & Shanghai, Zǒ-Sè Astrograph & 1983.05 & 29.89 & no filter & $B \approx 15$ & astr & \\
\hline S 83002 & Shanghai, Zǒ-Sè Astrograph & 1983.05 & 29.89 & no filter & $B \approx 15$ & astr & \\
\hline S 86006 & Shanghai, Zǒ-Sè Astrograph & 1986.04 & 29.89 & no filter & $B \approx 15$ & astr & \\
\hline S 86007 & Shanghai, Zǒ-Sè Astrograph & 1986.13 & 29.89 & no filter & $B \approx 15$ & astr & \\
\hline CCD pseudo-plate & Calar Alto, CAHA+OAN CCD & 1994.50 & 21.82 & $U B V R I$ & $V \approx 21$ & astr & $\mathrm{e}$ \\
\hline
\end{tabular}

Notes:

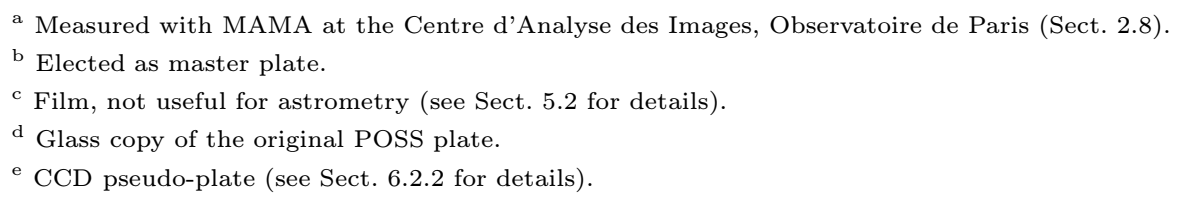

The present photographic study is based on astrograph and Schmidt plates from very different origins, spanning a large time baseline. In our effort to push the material to its limit of precision, we have done a careful treatment of the plate-to-plate geometric transformations, including a rigorous correction of magnitude effects. To this end, the joining of photographic and CCD data has been extremely useful. For the determination of proper motions, we implemented a version of the iterative central overlap algorithm (ICOA).
Our photographic photometry is derived from a set of modern epoch Schmidt plates, obtained with filter/emulsion combinations specifically designed to match Johnson's $B, V$ and $R$ bands. The density of stellar images on the plates was transformed into magnitude using the CCD photometry from Paper I as standard, and including colour terms in the transformation equations. This provided us with a complete and homogeneous deep photometric survey in the whole studied area.

This paper is organized into 10 main sections. In Sect. 2 we describe the plate material and its measurement. 
Section 3 is devoted to the photometric calibration of the plates, and includes a brief discussion of the resulting standard $B V R$ photographic photometry. The iterative central overlap algorithm is described in Sect. 4. Section 5 discusses some details on the plate-to-plate transformations. Section 6 is dedicated to our treatment of magnitude effects. The proper motion calculations are described in Sect. 7. The resulting photometric and astrometric photographic catalogue is presented and discussed in Sect. 8. A preliminary analysis of the data applying spatial distribution criteria is presented in Sect. 9. The conclusions are summarized in Sect. 10.

\section{Plate material and measurements}

We performed an exhaustive search for old plates containing the region of interest, and several modern epoch ones were obtained specifically for this work. All plates put at our disposal by several observatories were checked carefully, and those of good quality and covering the cluster area were selected. A total number of 29 photographic plates were used in this work. The maximum time difference among them is 103 years. The maximum difference in epoch among deep plates is 39 years. Table 1 lists the material used and the most relevant related data. Further information about each plate group is given in the following paragraphs.

\subsection{Modern epoch plates: $C A H A$ and $O C A$}

A set of six modern epoch Schmidt plates were obtained specifically for this work. Three of them were taken at Centro Astronómico Hispano-Alemán (CAHA, Calar Alto, Almería, Spain), and the other three at Côte d'Azur Observatory (OCA, CERGA, France). Table 2 summarizes some data about these plates. All of them were well centered in the clusters area. The scanned zone of these plates was a rectangle with center at the star PPM 94015, covering $12 \mathrm{~cm} \times 16 \mathrm{~cm}\left(2.9^{\circ} \times 3.8^{\circ}, \alpha \times \delta\right)$ in CAHA plates, and $13 \mathrm{~cm} \times 13 \mathrm{~cm}\left(2.4^{\circ} \times 2.4^{\circ}\right)$ in OCA plates. The filter-emulsion combinations permit a good reproduction of Johnson's $B, V$ and $R$ bands.

CAHA plates were kindly exposed and developed by J. Zamorano and Ó. Alonso (A 550), and by K. Birkle (A 573 and A 575). They are stored at the Departament d'Astronomia i Meteorologia of the Universitat de Barcelona.

OCA plates were obtained at CERGA Schmidt camera to the request of J. Guibert. They are stored at the clichothèque of the Centre d'Analyse des Images (Observatoire de Paris). Côte d'Azur plate OCA 3305 showed to be the deepest and finest from the astrometric and photometric points of view among our whole set of plates and, thus, it was selected as master plate for
Table 2. Modern epoch plates: Centro Astronómico HispanoAlemán (A) and Observatoire de la Côte d'Azur (OCA)

\begin{tabular}{lll}
\hline plate & center $\left(\alpha_{2000}, \delta_{2000}\right)$ & emulsion / filter \\
\hline A 550 & $5^{\mathrm{h}} 4^{\mathrm{m}} 34^{\mathrm{s}} 0+23^{\circ} 38^{\prime} 58^{\prime \prime}$ & IIIaJ/GG 385 \\
A 573 & $5^{\mathrm{h}} 4^{\mathrm{m}} 52^{\mathrm{s}} .2+23^{\circ} 39^{\prime} 24^{\prime \prime}$ & IIIaF/RG 630 \\
A 575 & $5^{\mathrm{h}} 4^{\mathrm{m}} 52^{\mathrm{s}} .2+23^{\circ} 39^{\prime} 24^{\prime \prime}$ & IIIaF/GG 475+BG 18 \\
OCA 3305 & $5^{\mathrm{h}} 1^{\mathrm{m}} 30^{\mathrm{s}} 0+23^{\circ} 30^{\prime} 00^{\prime \prime}$ & IIIaF/RG 610 \\
OCA 3308 & $5^{\mathrm{h}} 4^{\mathrm{m}} 00^{\mathrm{s}} 0+23^{\circ} 30^{\prime} 00^{\prime \prime}$ & IIaD/GG 495 \\
OCA 3314 & $5^{\mathrm{h}} 1^{\mathrm{m}} 30^{\mathrm{s}} 0+23^{\circ} 25^{\prime} 00^{\prime \prime}$ & TP 4415/RG 610 \\
\hline
\end{tabular}

${ }^{\text {a }}$ Film-based.

our ICOA implementation. The plate OCA 3314 is filmbased. In Sect. 5.2 we discuss the limits of its astrometric performance compared to classical glass plates.

\subsection{Palomar Observatory Sky Survey glass copies}

The studied area is contained in POSS I field number 1461. POSS plates 1461 in the $O$ (blue) and $E$ (red) bands were obtained in 1955 and are very deep. It has been shown that good quality copies of POSS plates are capable of giving good astrometric accuracy (Hunstead 1973). Our glass copies of POSS $1461 O$ and $E$ plates are very clean (no scratches nor drops are apparent), the emulsion and developing seem very uniform and the photometric calibration of the density of stellar images (Sect. 3) shows that the original dynamic range and limiting magnitude have been well preserved.

Both plates are centered at the position $\alpha_{2000}=$ $4^{\mathrm{h}} 54^{\mathrm{m}} 49^{\mathrm{s}}, \delta_{2000}=24^{\circ} 14^{\prime} 9^{\prime \prime}$. The scanned area was a rectangle of $10 \mathrm{~cm} \times 13 \mathrm{~cm}\left(1.9^{\circ} \times 2.4^{\circ}, \alpha \times \delta\right)$ centered on the star PPM 94015. The filter-emulsion combination of POSS plates are similar to Johnson's $B$ and $R$.

\subsection{Tautenburg plates}

Thanks to the proximity of the cluster zone to the Ecliptic, there exists a certain amount of old plates of NGC 1750 area obtained with the purpose of measuring minor planet positions. We have been able to locate and measure some of these plates from Tautenburg and Heidelberg (Sect. 2.4) observatories.

The plate files of the Astrophysical Observatory of Potsdam (Germany) were made available for this study by E. Schilbach. Four Schmidt plates taken with the big Tautenburg Schmidt camera were selected: two at epoch 1961 (T 109 and T 111), and two at epoch 1987 ( $\mathrm{T} 6573$ and $\mathrm{T}$ 6574). The older pair is centered at $\alpha_{2000}=5^{\mathrm{h}} 8^{\mathrm{m}} 20.9^{\mathrm{s}}, \delta_{2000}=+24^{\circ} 11^{\prime} 50^{\prime \prime}$, while the center for the other pair is $\alpha_{2000}=5^{\mathrm{h}} 8^{\mathrm{m}} 0.6^{\mathrm{s}}, \delta_{2000}=+24^{\circ} 15^{\prime} 45^{\prime \prime}$. In all cases, the scanned area was a rectangle of $12 \mathrm{~cm} \times$ $15 \mathrm{~cm}\left(1.7^{\circ} \times 2.1^{\circ}, \alpha \times \delta\right)$ around the star PPM 94048.

A slight elongation of stellar images was seen on some Tautenburg plates, probably due to guiding errors. 


\subsection{Heidelberg plates}

Plates from Heidelberg Observatory (Germany) were obtained with the Bruce Astrograph. They were made available for this study through the courtesy of S. Röser. The plate scale is not very favourable, because of the short focal length $(2 \mathrm{~m})$ of the instrument, but the epochs are old, and this led us to include this material in our study.

Heidelberg files contain a number of ancient plates centered near the Ecliptic in Taurus. Four of them were selected because they contained the whole cluster area, and their image quality was good: B 1118 (epoch 1904), B 1386 (epoch 1905), B 3563 (epoch 1914) and B 4191 (epoch 1919). Unfortunately, huge problems arose for the measurement of several of these plates. Probably due to glass bending, B 1386 and B 3563 led to serious focusing difficulties in the device, and it was not possible to obtain useful data from them.

Plates B 1118 and B 4191 were successfully measured. The first one was not only the oldest, but also the deepest and finest in image quality among the four Bruce plates. B 1118 is centered at $\alpha_{2000}=$ $5^{\mathrm{h}} 8^{\mathrm{m}} 59.9^{\mathrm{s}}, \delta_{2000}=+24^{\circ} 58^{\prime} 47^{\prime \prime}$. B 4191 is centered at $\alpha_{2000}=4^{\mathrm{h}} 57^{\mathrm{m}} 59.6^{\mathrm{s}}, \delta_{2000}=+25^{\circ} 2^{\prime} 34^{\prime \prime}$. The measured zone was a square of $8.5 \mathrm{~cm} \times 8.5 \mathrm{~cm}\left(2.4^{\circ} \times 2.4^{\circ}\right)$ around the star PPM 94013 in both cases.

\subsection{Astrographic Catalog plates}

The Astrographic Catalog has been re-reduced and published at the United States Naval Observatory (Urban \& Corbin 1996; Urban 1997). They used the original plate measurements and determined new plate constants taking modern catalogues as reference. In spite of having been manually measured, the original $\mathrm{AC}$ data have shown to be useful and of good quality. Recently, the combination of AC positions with Tycho catalogue (ESA 1997) allowed a noticeable improvement of Tycho proper motions (Urban et al. 1997).

S.E. Urban provided us with the files containing the original $\mathrm{AC}$ positional and photometric manual measurements from the individual AC plates close to the cluster area. Five AC plates from Paris zone were very suitable for our work. One of them (AC 2338) is almost centered on the clusters, while four additional plates (AC 2238, AC 2239, AC 2438, AC 2439) overlap with the former in a corner-on-center disposition. Each plate covers a field of view of $160^{\prime} \times 160^{\prime}$. These data are the oldest ones used in our proper motion determination (epochs from 1891 to 1895, see Table 1). The absolute accuracy of the original positions in Paris zone is reported to be of $\sigma_{\alpha}=0.26^{\prime \prime}, \sigma_{\delta}=0.20^{\prime \prime}$ by Eichhorn (1974), and $\sigma_{\alpha}=0.22^{\prime \prime}, \sigma_{\delta}=0.21^{\prime \prime}$ by Urban (1997).

\subsection{Shanghai plates}

A total number of ten plates from Zǒ-Sè Astrograph (Shanghai, China) were used in our study. These plates are $24 \mathrm{~cm} \times 30 \mathrm{~cm}$ in size, which corresponds to $2.0^{\circ} \times 2.5^{\circ}$ with the plate scale of the instrument $\left(29.89^{\prime \prime} \mathrm{mm}^{-1}\right)$. All of them were well centered in the cluster region and nonfiltered. The old epoch plates (1918 and 1930) were used by $\mathrm{Li}(1954)$ in his proper motion determination in the NGC 1750 area.

All ten Shanghai plates were measured with a PDS machine in the Dominion Astrophysical Observatory (Canada) and the measurements were supplied for the present work by two of us (K.P.T. and J.L.Z.). The files contained, for each star, the sequence number of the measurement, star coordinates, their estimated errors and the densities of the star images (in the form of instrumental magnitude). The estimated errors in the positions are usually around or below $1 \mu \mathrm{m}$ for well measured stars.

A total of 15 stars were re-measured three times along the plate scan process, in order to monitor possible drifts. These "drift stars" were measured also as program stars. So, a total of four measurements were available for each drift star on each plate. Although the output data do not contain time information, the sequence number of the measurements can be used as a time indicator. For each drift star, taking one position as reference and plotting the coordinates change as a function of the sequence number, slight drifts were detected in some cases. Although most of these drifts were below the formal accuracy of the measurements, in several plates the tendency was so clear that it seemed convenient to apply a linear correction.

\subsection{CCD pseudo-plate}

The whole set of CCD fields from Paper I was assembled into a CCD pseudo-plate. This plate should not be confused with the "global CCD frame" described in Paper I. That global frame was composed by simple transformation of contiguous fields using the stars in the overlap areas, and was used for computing approximate equatorial coordinates for the photometric catalogue in the FK5 reference system.

But the present CCD pseudo-plate is produced in a more sophisticated way, avoiding the intrinsic inaccuracies of tranformations through slight overlaps. Because of its deep link to the treatment of inter-plate magnitude equation, we describe the construction and properties of the CCD pseudo-plate in Sect. 6.2.2.

\subsection{Plate measurements with MAMA}

As mentioned above, plates from CAHA, OCA, POSS, Heidelberg and Tautenburg were measured specifically for this study at the Centre d'Analyse des Images at 
the Observatoire de Paris, using the high precision microdensitometer "Machine Automatique à Mesurer pour l'Astronomie" (MAMA $\left.{ }^{1}\right)$.

This device has a superb optical and mechanical performance (Guibert et al. 1990). It uses a quartz-iodine illuminating source, whose transmitted light is detected by a reticon, 1024 pixels large, with a pixel size of $10 \mu \mathrm{m}$. The plate is moved under the light source during the scanning process. Our plates were scanned in the smallest pixel mode, $10 \mu \mathrm{m}$. The repeatability of the measurements with the MAMA is around of $0.2 \mu \mathrm{m}$, and the absolute accuracy of the measurements is $0.6 \mu \mathrm{m}$ (Soubiran 1992).

MAMA can operate in two measuring modes (Berger et al. 1991): pavé and zone. In the pavé mode, an input list of program objects is provided to the machine, and it scans a small plate area around the expected position of each of them. In the zone mode, an extended, continuous region of the plate is specified and scanned. In our case, the pavé mode was used for the scanning of a list of photometric standard stars (Sect. 3.1) inside the clusters area, and also for recording the PPM (Röser \& Bastian 1989) stars present on the whole plate with the aim of performing a preliminary astrometric reduction. The plate areas specified in previous subsections for each plate were scanned in zone mode.

In pavé as well as in zone mode, the resulting digital images were treated with a multi-threshold objectdetection and reconstruction algorithm. A maximum number of 9 thresholds was used for our plates. The lowest threshold was chosen to be 3 times the standard deviation of the sky background. For each density threshold, the algorithm produces an output list of detected objects with photocenter position, object area, integrated flux density and various morphological parameters. The flux determinations are automatically corrected for the local sky background. A second algorithm applied to the output files performs the cross-identification and grouping of data for each object detected on different thresholds. In this step, an identification number is assigned to each individual object.

In order to avoid noise in the data files, objects detected only at the lowest thresholds (1 and/or 2) were suppressed. Since the object position at each threshold is identified with the photocenter, the object area has to be large enough to provide a good accuracy. Following Soubiran (1992), we ignored all entries having an area smaller than 10 pixels.

The pavé mode detection of PPM stars on the whole plate allows to determine the object positions in the FK5 reference system. In this way, for each scanned plate we got a preliminary astrometric catalogue that allowed us to perform initial cross-identifications between different plates (Sect. 4).

1 MAMA (Machine Automatique à Mesurer pour l'Astronomie) was developed and is operated by INSU/CNRS.

\section{Photographic photometry}

\subsection{Transformation to the standard photometric system}

Photographic photometry was obtained from several modern epoch (filtered) plates by calibrating the photographic flux using the $B V R-\mathrm{CCD}$ observations from Paper I as reference. A catalogue of more than 400 photometric secondary standards was prepared following these criteria:

1. The photometric standards should be well isolated, in order to avoid the risk of misidentification when targeting MAMA in pavé mode;

2. The photometric standards should be well distributed along the whole magnitude interval covered by the deep plates, avoiding an excessive presence of faint stars.

Standard magnitudes from Paper I and logarithm of photographic flux were related by fitting a polynomial with a linear colour term. Since our deepest plates are in $R$ and $B$ bands, we used the colour index $B-R$ for the transformation equations:

$B=b_{0}(B-R)+\sum_{i=1}^{k} b_{i}\left(\log _{10} \phi_{B}\right)^{i-1}$

$V=v_{0}(B-R)+\sum_{i=1}^{m} v_{i}\left(\log _{10} \phi_{V}\right)^{i-1}$

$R=r_{0}(B-R)+\sum_{i=1}^{n} r_{i}\left(\log _{10} \phi_{R}\right)^{i-1}$

where $\phi_{X}$ represents the stellar flux measured at one threshold in a $X$-band plate; $B, V$ and $R$ are the standard Johnson-Cousins magnitudes; $b_{i}, v_{i}$ and $r_{i}$ are the transformation coefficients, and $k-1, m-1$ and $n-1$ are the polynomial degrees. Each filtered plate was calibrated in the band closest to that defined by its filter/emulsion combination (Table 1). The coefficients in Eqs. (1) to (3) were obtained by a least squares method for each plate and threshold. The polynomial degree, from 3 rd to 5 th, was selected depending on the plate response curve, and was the same for every threshold on each plate. Table 3 displays some data about the standard photometric fits: polynomial degree, number of thresholds $\left(N_{\mathrm{thr}}\right)$ and standard deviation of the residuals of the fits, averaged among all thresholds $(\langle\sigma\rangle)$. Regarding the goodness of the fits and the magnitude intervals covered, we decided to use, for the final photographic photometry, the following plates: A 550 for $B$ magnitude; A 575 for $V$ magnitude; OCA 3305 and OCA 3314 for $R$ magnitude. As an example, Fig. 1 shows the relations among standard magnitude and logarithm of flux for one threshold of each selected photometric plate.

In order to apply the above transformations to nonstandard stars, a cross-identification among the different photometric plates is needed. The standard magnitudes are calculated, for a given star, following these steps: 

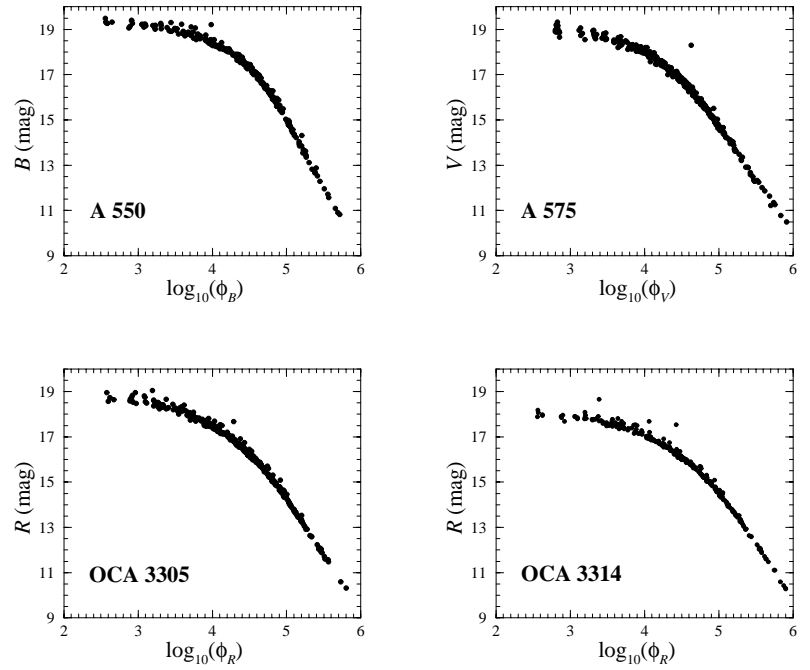

Fig. 1. Relation among logarithm of flux and standard magnitude for the third threshold of each plate selected for deriving photographic photometry

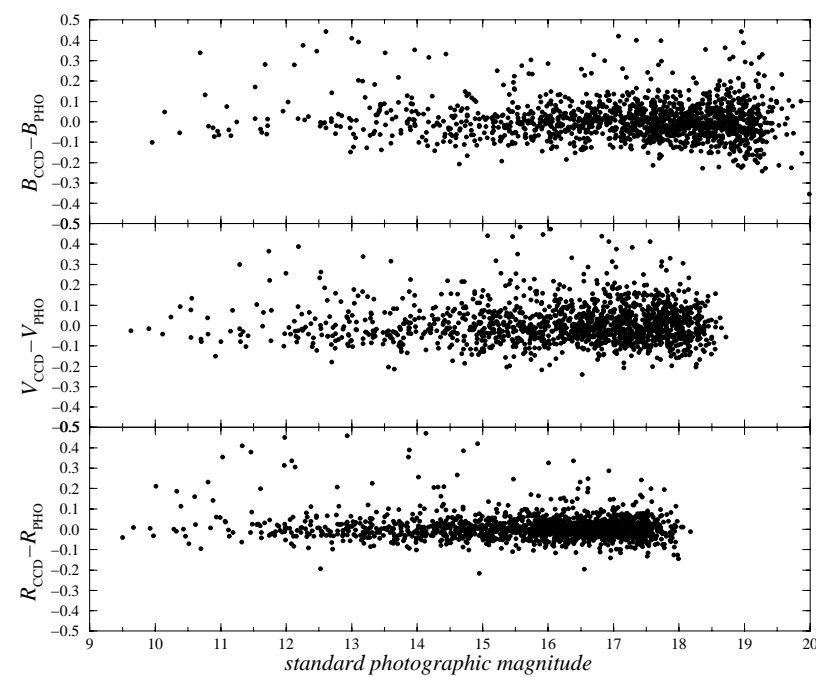

Fig. 2. Comparison of photographic photometry with the whole CCD photometric sample from Paper I. The panels, from top to bottom, show the differences CCD-photographic in $B(1477$ stars), $V$ (1498 stars) and $R$ (1536 stars) bands.

Table 3. Photographic photometry: standard deviation of the residuals of the standard stars $(\langle\sigma\rangle)$ averaged for all the thresholds on each filtered plate. $N_{\text {thr }}$ gives the number of thresholds and "Degree" is the polynomial degree of the fit

\begin{tabular}{lcccc}
\hline Plate & Band & Degree & $N_{\text {thr }}$ & $\langle\sigma\rangle$ (mag) \\
\hline A 550 & $B$ & 5 & 9 & 0.07 \\
A 573 & $R$ & 5 & 7 & 0.08 \\
A 575 & $V$ & 5 & 9 & 0.11 \\
OCA 3305 & $R$ & 5 & 9 & 0.06 \\
OCA 3308 & $V$ & 3 & 7 & 0.12 \\
OCA 3314 & $R$ & 5 & 9 & 0.06 \\
POSS 1461-O & $B$ & 5 & 9 & 0.14 \\
POSS 1461-E & $R$ & 5 & 8 & 0.12 \\
\hline
\end{tabular}

First, as can be easily deduced from Eqs. (1) and (3), the standard $R$ magnitude can be computed from the expression

$R=\frac{r_{0} \sum_{i=1}^{k} b_{i}\left(\log _{10} \phi_{B}\right)^{i-1}+\left(1-b_{0}\right) \sum_{i=1}^{n} r_{i}\left(\log _{10} \phi_{R}\right)^{i-1}}{1-b_{0}+r_{0}}$.

For one star detected at a threshold on an $R$ photometric plate, we have a definite value of its flux $\phi_{R}$. But for obtaining its standard $R$ magnitude we need not only the coefficients $\left\{r_{i} ; i=0, \cdots, n\right\}$ for this specific threshold, but also one blue flux $\phi_{B}$ from one threshold on the $B$ plate, with the corresponding coefficients $\left\{b_{i} ; i=0, \cdots, k\right\}$. Our photometric procedure computes different $R$ values using all the $\phi_{B}$ fluxes (all thresholds) available from the $B$ plate (A 550) for this star, and averages the results using the standard deviation of the residuals of the standard stars in the individual blue fittings as a weight. The process is repeated for all the $R$ thresholds available for this star on this $R$ plate, and the different resulting $R$ values are averaged using the standard deviation of the residuals of the individual $R$ fittings as a weight. The standard deviation of this average is stored. This yields an $R$ standard magnitude for this star on this $R$ plate. The same procedure is followed on the other photometric $R$ plate, yielding another $R$ value with its standard deviation. The two resulting $R$ values are averaged using their standard deviations as weights.

The second step, the determination of standard $B$ magnitudes, is somewhat simpler, because only one photometric plate is used, and because the standard $R$ value has been already computed and can be used in the calculation. From Eqs. (1) and (3) we see that $B$ is obtained as:

$B=\frac{-b_{0} R+\sum_{i=1}^{k} b_{i}\left(\log _{10} \phi_{B}\right)^{i-1}}{1-b_{0}}$.

For each star detected on the $B$ photometric plate (A 550 ), one $B$ magnitude is computed from each threshold, and the resulting values are averaged using the standard deviation of the residuals of the standard stars at each threshold as a weight. In a third step, a similar process is applied for the calculation of $V$ from the $V$ photometric plate (A 575), using Eq. (2).

\subsection{The photographic photometric catalogue}

The final photometric catalogue (Table 6, available only in electronic form) contains a total number of 39762 stars, 38304 having complete $B V R$ photographic photometry, and 1458 only $B R$ photographic photometry. The stars are designated by their identification number on the master plate OCA 3305. Table 4 gives the number of stars by interval of magnitude in each band.

The absolute accuracy of the photographic photometry is limited by several factors. One of them is the emulsion and developing uniformity. Since our photometric standards are located in the center of the scanned zones, the 
Table 4. Photographic photometry: total number of stars $(N)$ in the photographic photometric catalogue and standard deviation $(\sigma)$ of the differences with CCD photometry for the stars in common, in magnitude intervals

\begin{tabular}{|c|c|c|c|c|c|c|}
\hline \multirow{2}{*}{$\begin{array}{l}\text { mag. } \\
\text { range }\end{array}$} & \multicolumn{2}{|c|}{$B$} & \multicolumn{2}{|c|}{$\bar{V}$} & \multicolumn{2}{|c|}{$R$} \\
\hline & $N$ & $\bar{\sigma}$ & $N$ & $\bar{\sigma}$ & $N$ & $\bar{\sigma}$ \\
\hline$<10$ & & & $\overline{5}$ & 0.07 & $\overline{43}$ & 0.10 \\
\hline $10-11$ & 31 & 0.11 & 97 & 0.12 & 171 & 0.13 \\
\hline $11-12$ & 106 & 0.14 & 217 & 0.07 & 391 & 0.04 \\
\hline $12-13$ & 223 & 0.13 & 533 & 0.08 & 796 & 0.04 \\
\hline $13-14$ & 509 & 0.08 & 1073 & 0.08 & 1570 & 0.04 \\
\hline $14-15$ & 993 & 0.07 & 2068 & 0.08 & 3268 & 0.04 \\
\hline $15-16$ & 2029 & 0.07 & 4010 & 0.08 & 5844 & 0.04 \\
\hline $16-17$ & 3953 & 0.07 & 7010 & 0.08 & 9887 & 0.04 \\
\hline $17-18$ & 7202 & 0.07 & 10719 & 0.08 & 13424 & 0.04 \\
\hline $18-19$ & 11412 & 0.08 & 11883 & 0.08 & 4368 & 0.05 \\
\hline $19-20$ & 13020 & 0.09 & 689 & 0.08 & & \\
\hline $20-21$ & 284 & 0.10 & & & & \\
\hline TOTAL: & 39762 & 0.08 & 38304 & 0.08 & 39762 & 0.04 \\
\hline
\end{tabular}

photometric calibration would be strictly valid only in this area. But we extrapolated the calibrations and applied them to the whole $2.3^{\circ} \times 2.3^{\circ}$ region. We have no means of evaluating the possible existence of photometric inhomogeneities on the photometric plates, but visual inspection of them shows an apparent good regularity of emulsions and developing.

Another source of error arises from the quality of the original CCD photometry used for the calibration. We have no reason to suspect systematic effects in the CCD data. We refer to Paper I for a detailed discussion of them.

Further uncertainties may be caused by the transformation from the effective band defined by the emulsion/filter combinations to the standard system. This effect is taken into account to some extent by means of the colour term introduced in the transformation equations. The effect of the colour term coefficients was small in all cases, and introducing further colour terms (quadratic, and so on) in the transformation equations did not improve the goodness of the fits.

The non-linearity of the photographic response is well corrected by using the appropriate polynomial degree in the transformation. The ultimate limit of accuracy lies in the intrinsic precision of the photographic emulsion. For these reasons, it is usually difficult to reach precisions better than $0.1 \mathrm{mag}$ in photographic photometry. The average standard deviations of the residuals of the photometric fits (Table 3) seem to indicate that we have reached, or even improved, this limit.

In order to estimate the quality of our photographic photometry as a function of magnitude, we compared the obtained $B V R$ values with the whole photometric catalogue from Paper I. As explained, a subsample of around 400 of these stars were used as secondary standard for the calibration, but the comparison with the whole set allows a better determination of the photometric uncertainties as a function of magnitude. Figure 2 displays the results of the comparison. Table 4 includes the standard deviations of the differences $C C D$-photographic in one magnitude bins and applying a $3 \sigma$ clipping to the data in each in- terval. The differences contain contributions as from photographic and from CCD photometric errors, but the second are small compared to the first ones.

\subsection{Raw photographic photometry for cross- identifications}

As explained in Sect. 4.1, the cross-identification step of the plate-to-plate transformation algorithm applies a double position-brightness criterion for matching the stars and, thus, it requires some kind of brightness estimator for every object in each plate, not only in filtered ones.

Just for cross-identification purposes, for every plate and threshold we performed a least squares fit of magnitude as a function of logarithm of flux without colour terms. The fitted model was a polynomial, and the standard stars were those used in Sect. 3.1. These polynomials were applied to all the detected objects and the different magnitudes coming from different thresholds in the same plate were averaged using as weight the object area at each threshold.

The reference bands for these raw magnitude calibrations were selected in accordance with each plate/emulsion characteristics. Johnson's $B$ magnitude was used for nonfiltered plates (Tautenburg, Heidelberg) and for $B$-like filtered plates (A 550, POSS 1461-O). For AC plates, the photographic magnitude values quoted in the original plate measurements were used directly. The instrumental PDS magnitudes from Shanghai plates were transformed into Johnson's $B$ magnitude fitting a 2nd degree polynomial using the $B-\mathrm{CCD}$ measurements as reference. Although our master plate (OCA 3305) matches the $R$ band, we performed a raw calibration also into Johnson's $B$ filter, in order to allow the cross-identification with $B$ like plates.

$R$-like plates (the master plate OCA 3305 , OCA 3314 , A 573 and POSS $1461-\mathrm{E}$ ) were fitted in a similar way to the standard Cousins' $R$ band. $V$-like plates (OCA 3308 and A 575) were fitted, for comparison purposes, with Cousins' $R$ band, and this raw $R$ magnitude was used to perform their cross-identification with the master plate. When there was cross-identification of the CCD pseudoplate with the master plate, the $R$ magnitude from Paper I was compared to the raw $R$ magnitude computed in the master plate.

Several stars were saturated in some or all plates. These objects were maintained in the files, labelled as "bright", and they received a special treatment in the cross-identification procedures.

\section{Iterative central overlap algorithm}

There are two different widespread approaches to the calculation of proper motions from photographic positions: 
the plate pairs method and the central overlap method. In the plate pairs technique, all plates are transformed into a common reference frame and, after that, they are paired following some reasonable criteria. Usually, pairs are constructed with plates spanning a wide time baseline and, if possible, having similar accuracies, limiting magnitudes and filters. For each star, the proper motion is calculated once in each plate pair, and the different determinations obtained are averaged. The plate pairs method performs best when the plate material is homogeneous (Schilbach et al. 1995; Tian et al. 1994; Zhao \& He 1990).

The central overlap technique was first proposed by Eichhorn \& Jefferys (1971). It intends to determine the plate-to-plate transformation parameters, the stars motions and the errors simultaneously, using all the data at the same time. This method has rigorous mathematical foundations (Eichhorn 1988), but its computational requirements are so huge that, in practice, it cannot be implemented in its strict formulation. The usual approach to the method is generally known as iterative central-overlap algorithm (ICOA), and implies the separation of the determination of plate and star parameters in consecutive steps that are iterated until convergence is achieved. This procedure is known to be equivalent, in practice, to the one-step block-adjustment approach (Tucholke 1992), and has been extensively used during the last decades (for instance, Cudworth et al. 1993; Van Altena et al. 1988; Tucholke et al. 1994).

Given the diversity of the plate material used in this study, we decided to use an ICOA for our astrometric analysis. Our implementation, similar to that by Jones \& Walker (1988), is summarized in the flow chart of Fig. 3. One plate is selected as reference ("master" plate). All the other plates ("source" plates) are tied into the master reference system. Proper motions are computed from different positions at different epochs, and they are fed-back to improve the transformations of source plates to the master plate reference system. The whole process is iterated until the computed proper motions converge. The best modern epoch plate, OCA 3305, was selected as master.

To begin the process, an initial input list with a number of stars cross-identified with the master plate is needed for each source plate. These initial cross-identification files were generated by matching the equatorial coordinates contained in the preliminary astrometric catalogue of each plate (Sect. 2.8).

\subsection{The transformation-crossing loop}

The transformation-crossing loop was applied in turn, one by one, to all plate files. This essential piece of the algorithm consists of two steps: the computation of transformation equations from source plates to the master plate, and the application of these equations to generate crossidentification lists as complete as possible.
The code calculates the transformation coefficients by a least squares fit, with $3 \sigma$ clipping: the fit is performed iteratively, discarding at each step those stars whose residual is larger than $3 \sigma$, until no more stars are eliminated.

The program admits a general plate model of the following form:

$x^{\prime}=P_{n, x}(x, y)+Q_{x}(x, y, m) ;$

$y^{\prime}=P_{n, y}(x, y)+Q_{y}(x, y, m)$.

In these equations, $\left(x^{\prime}, y^{\prime}\right)$ are the coordinates on the master reference system; $(x, y)$ are the coordinates on the source plate system; $m$ is the apparent magnitude, and $P_{n, x}(x, y), P_{n, y}(x, y)$ are complete two-dimensional polynomials of $n$-th degree. For instance,

$P_{2, x}(x, y)=a_{0}+a_{1} x+a_{2} y+a_{3} x^{2}+a_{4} x y+a_{5} y^{2}$.

$Q_{x}(x, y, m)$ and $Q_{y}(x, y, m)$ are polynomial functions containing some magnitude-dependent terms.

So, the general model has a purely geometrical part, beside a mixed geometrical-photometric dependence. The specific plate models used in the calculations will be discussed in Sects. 5 and 6.

The cross-identification routine applies the just computed transformation equations to all stars in the source plate and performs a complete cross-identification with the master plate. The cross-identification takes into account a double position-brightness criterion:

1. For a given master plate star, all the stars in the source plate within a specified search radius are candidates to be cross-identified;

2. Among the candidates, those stars whose magnitude difference with the master plate star is larger than a specified magnitude tolerance are eliminated, and

3. From the remaining candidates, the spatially closest is selected.

The search radius and magnitude tolerance are parameters set up for each plate. The search radius is a function of the epoch difference with the master plate. For contemporary plates, a search radius of $5^{\prime \prime}$ was selected. The search radius grows linearly with time in such a way that for an epoch difference of 90 years, it becomes $9^{\prime \prime}$.

The magnitude difference tolerance was set to $3 \sqrt{\sigma_{\text {master }}^{2}+\sigma^{2}}$, where $\sigma_{\text {master }}$ and $\sigma$ are the dispersions of the fit performed on the master and source plates for cross-identification purposes (Sect. 3.3), respectively.

The resulting list of cross-identifications was reintroduced into the first element of the loop, in order to compute an improved plate-to-plate transformation. The loop was iterated until the number of cross-identifications in the output list stabilized. This resulted in one file for each source plate, containing the positions of all matched objects for the epoch of the source plate in the reference system of the master plate.

The programs used in the transformation-crossing loop were adapted from $C$-codes kindly provided by C. Alard, and originally designed for the DUO (Disk Unseen Objects) project (Alard \& Guibert 1997). 


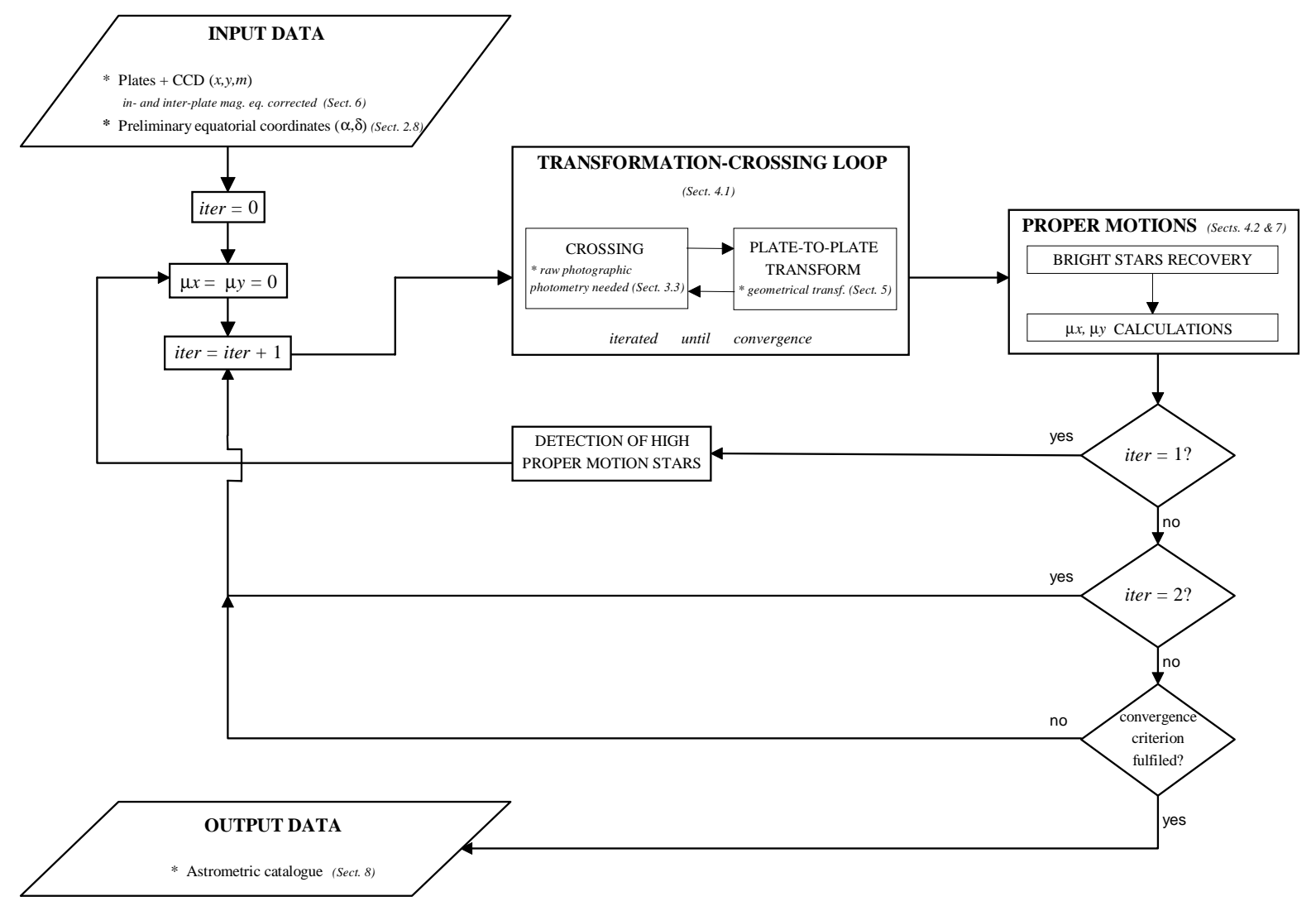

Fig. 3. Flow chart of the iterative central overlap algorithm (ICOA) used for the determination of proper motions

\subsection{Calculation of proper motions}

The set of output plate files in the master plate reference system were compiled. The resulting table was scanned in order to detect non cross-identified bright stars, for which it was impossible to compute a magnitude estimator for cross-identification (Sect. 3.3). Since the raw magnitudes used for cross-identification purposes were in some cases computed by fitting the photographic flux to a filter not similar to the band determined by the plate filter/emulsion, stars with very red or very blue colours could result unmatched. For this reason, the recovery of bright stars extended not only to saturated stars, but also to other unmatched bright stars (down to $V \approx 13 \mathrm{mag}$ ).

After the automated recovery of unmatched bright stars, proper motions were computed in the master plate reference system by means of least squares fits of position as a function of epoch:

$$
\begin{aligned}
& x_{i, p}-x_{i, \text { master }}=\xi_{i}+\mu_{i, x}\left(t_{p}-t_{\text {master }}\right) ; \\
& y_{i, p}-y_{i, \text { master }}=\eta_{i}+\mu_{i, y}\left(t_{p}-t_{\text {master }}\right) .
\end{aligned}
$$

In these equations, $\left(x_{i, p}, y_{i, p}\right)$ are the coordinates for star $i$, measured on plate $p$ and transformed to the master plate reference system. $\left(x_{i \text {,master }}, y_{i \text {,master }}\right)$ are the coordinates for the same star, measured on the master plate. $t_{p}$ and $t_{\text {master }}$ are the epochs of plate $p$ and the master plate, respectively. The calculation was performed only for stars having positions spanning a minimum epoch interval of $30 \mathrm{yr}$. The fitted parameters are $\left(\xi_{i}, \mu_{i, x} ; \eta_{i}, \mu_{i, y}\right)$. $\mu_{i, x}$ and $\mu_{i, y}$ are the proper motions. $\xi_{i}$ and $\eta_{i}$ should be understood (Jones \& Walker 1988) as corrections to the master plate measured positions.

The index $p$ run over the whole plate set, including the master plate. The fits were performed including a weighting system that took into account the plate scale (Sect. 7.1). The proper motions fits included a $3 \sigma$ clipping. For a given star, after performing a proper motion calculation in both coordinates, the largest residual in $x$ and $y$ was checked. If one or both of them were larger than $3 \sigma$, the plate was removed from both fits for this star and the fit parameters re-computed. The checking and rejection was repeated until all the residuals were smaller than $3 \sigma$. The resulting parameters and standard errors were recorded.

\subsection{Second and further iterations}

High proper motion stars can distort the reference frame, if their motion is not taken into account in the plateto-plate transformations. At the end of the first iteration, a search for high proper motion stars was performed through the output list. The stars with modulus of proper motion larger than 6 mas $\mathrm{yr}^{-1}$ were labelled. All proper 


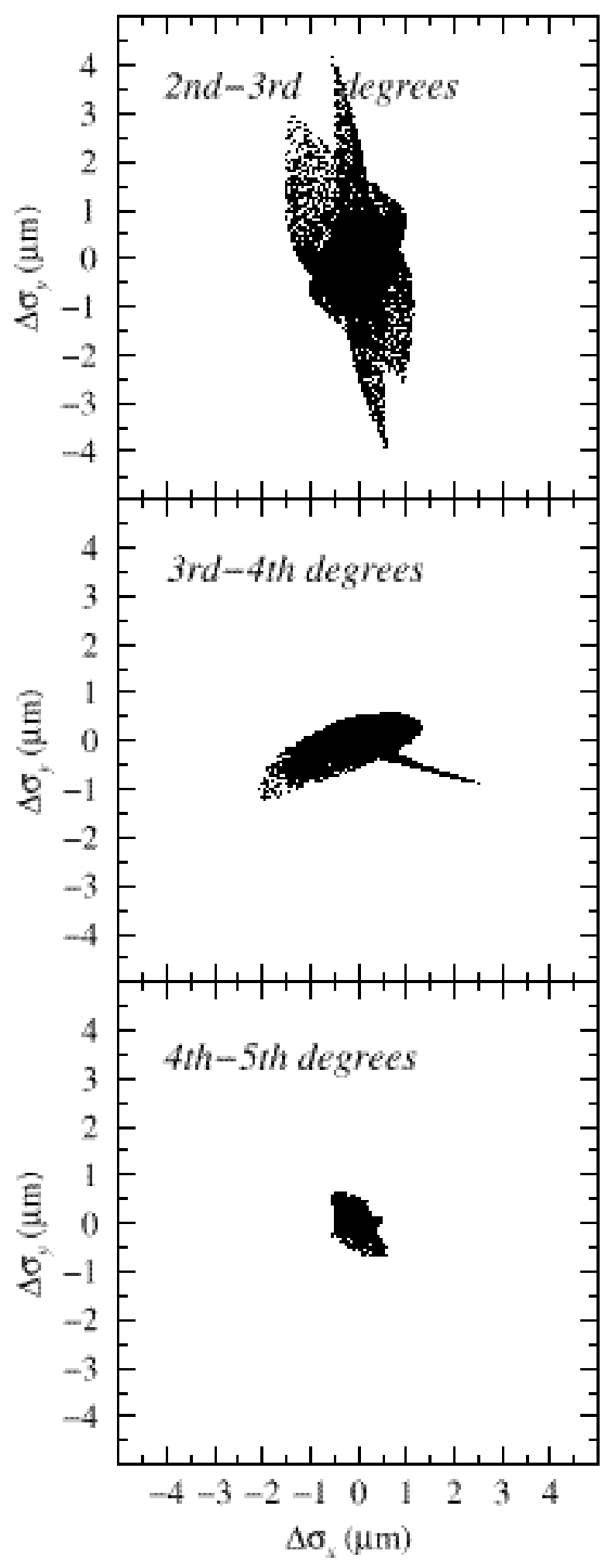

Fig. 4. Change in the residuals of the geometric transformation of the source plate A 550 to the master plate OCA 3305 for successive pairs of polynomial transformations with increasing degrees. The selected degree was $4^{\text {th }}$ motions were set again to zero, and the whole process was repeated. But now, in the second iteration, high proper motion stars were not used for computing the plate-toplate transformations. However, they were included in the last cross-identification performed in the transformationcrossing loop, and they continued the rest of this and further iterations as any other star.

From the third iteration on, positions of all stars in the master plate were converted to the epoch of each source plate, by applying the parameters $\left(\xi_{i}, \eta_{i}\right)$ and $\left(\mu_{i, x}, \mu_{i, y}\right)$, before entering into the transformation-crossing loop. At this stage, the reference frame is no longer linked only to the physical positions measured on the master plate, but depends on the whole set of astrometric data.

At the end of the third and following iterations, the mean change of the proper motions from the previous to the last iterations was computed, using the formal proper motion errors as a weight. When the average change was well below the mean error, the iterations stopped.

\section{Geometrical transformations}

\subsection{Polynomial degree of the transformations}

The polynomial degree of the plate models was selected individually for each source plate. The tested plate models were based on Eqs. (4) and (5), without magnitudedependent terms and without taking into account the proper motions. Since the expected density of cluster members in our field is small, ignoring proper motions and magnitude effects could introduce a larger dispersion (mainly in old epoch plates), but not global systematic effects.

We tried out different polynomial degrees, and we analyzed the residuals of the transformations by computing their difference among consecutive degrees. The $n$-th degree was accepted when the following two conditions were fulfilled:

1. The changes of the residuals from the $n$-th to the $(n+1)$-th degree are around or below $1 \mu \mathrm{m}$, and

2 . The residuals from the $n$-th degree fit do not show any spatial systematic behavior.

As an example of the assignation of transformation degrees, Fig. 4 shows the difference of residuals of the transformation among several consecutive degrees for one plate (A 550), and Fig. 5 displays the spatial pattern of the residuals for the same plate and the selected degree $\left(4^{\text {th }}\right)$, averaged in $1 \mathrm{~cm}$ squares. Usually, third or fourth degree was enough for accomplishing both required conditions.

\subsection{Anomalous plates}

While testing the degree for the transformation equations, the low astrometric quality of two plates became evident: 


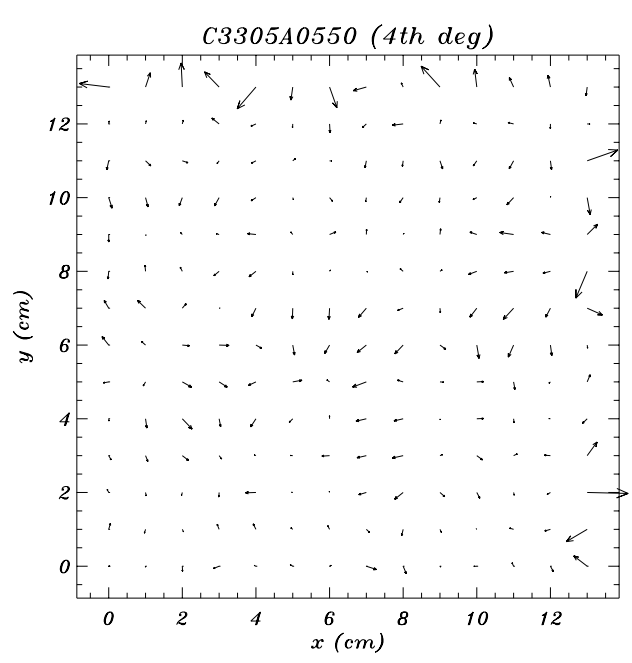

Fig. 5. Spatial behaviour of the residuals of the fourth degree transformation polynomial from the source plate A 550 to the master plate OCA 3305 . Each arrow represents the average residual in a $1 \mathrm{~cm} \times 1 \mathrm{~cm}$ square. An arrow with length equivalent to $1 \mathrm{~cm}$ represents a mean residual of $0.25 \mu \mathrm{m}$

A 575 and OCA 3314. The CAHA plate displayed a quite asymmetric systematic distribution of the residuals. These features were difficult to explain from usual optical or mechanical effects in Schmidt plates, and led to residuals of the order of $1 \mu \mathrm{m}$ and systematic trends even when applying a 5 -th degree polynomial. The reduction of the residuals required a very high degree (8-th or even more). We preferred to eliminate this plate for astrometric applications, although it was used as source of our $V$ photographic photometry.

OCA 3314 plate is film-based. With the stopping of glass-plate production, the use of film substrates for big format photographic emulsions is being considered a possible alternative, at least until CCD technology will be able to cover wide fields with enough astrometric quality. The inclusion of one film among our material provided a chance for testing the astrometric and photometric performance of this kind of detectors compared to classical glass plates.

The photometric accuracy of plate OCA 3314 is excellent. Its filter/emulsion combination (Table 2) matches $R$ band, and the emulsion sensitivity was good enough to yield really small residuals in the photographic calibration (Table 3). However, the astrometric transformation to the master plate system made evident the presence of a horizontal deformation pattern mixed up with some local systematics. Likely, this deformation pattern is present not only in our $13 \times 13 \mathrm{~cm}$ scanned area, but all over the plate. High degree polynomials reduced, but did not eliminate this pattern (Fig. 6) and some systematic trends still remained even with an 8th degree transformation.

We conclude that the film OCA 3314 has a general spatial deformation, in a 1-2 $\mathrm{cm}$ band pattern parallel to
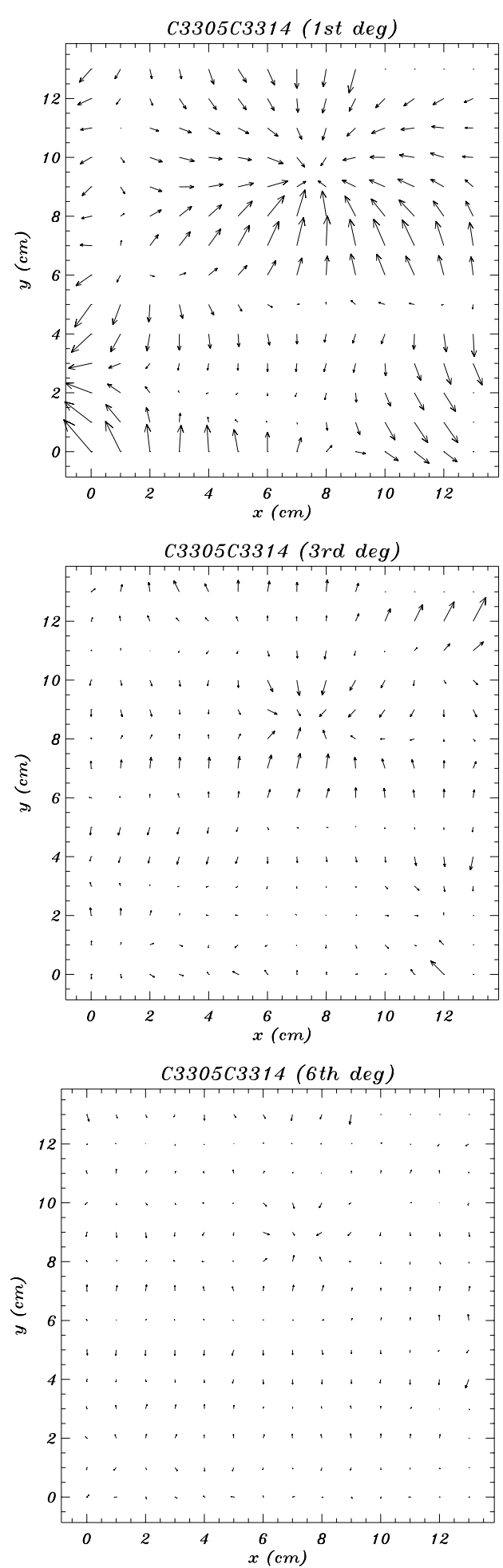

Fig. 6. Spatial behaviour of the residuals of the transformation from the film-based source plate OCA 3314 to the master plate OCA 3305. The plate models were complete 2-D polynomials of different degrees, without magnitude terms. Each arrow represents the average residual in a $1 \mathrm{~cm} \times 1 \mathrm{~cm}$ square. An arrow with length equivalent to $1 \mathrm{~cm}$ represents a mean residual of $1 \mu \mathrm{m}$ (this scale is four times bigger than in Fig. 5) 
$x$ axis, with some other local strong systematics (sink and source points). The systematic trends induced by this deformation are not easy to eliminate, and the best solution is to discard the film plate for astrometric purposes. Whether these conclusions could be extended to all filmbased plates or not, is a question worth of attention and should be investigated performing extensive tests with different kinds of film-based photographic plates.

\section{Magnitude effects}

As it is well known, the non-linear response of the detector, and/or an asymmetric point spread function (PSF), can introduce a magnitude-dependent shift in star positions recorded on two-dimensional detectors. Asymmetric PSF profiles can arise from several causes, the most usual being imperfect guiding. The non-linearity of photographic response makes the recorded PSF profile to be different for objects of different brightness. This effect, usually known as magnitude equation, induces magnitudedependent trends that can be propagated to the final proper motions (Dinescu et al. 1996) and causes serious biases in the analysis of the results, if it is not properly taken into account.

We call inter-plate magnitude equation the magnitude effects involved in plate-to-plate transformations. Our specific treatment is fully discussed in Sect. 6.2. Another kind of magnitude-dependent effect is present in our MAMA data, due to the interaction between asymmetric PSFs with the multi-thresholding processing of the images. This effect, which we call in-plate magnitude equation, is discussed in the next section.

\subsection{In-plate magnitude equation}

Let us consider an asymmetric PSF recorded on a photographic plate. When this PSF is sliced at different density thresholds, as was done in our MAMA measurements, the photocenters of the different cut profiles, in general, do not coincide. If a plate is affected by magnitude equation, the positional shift of the photocenters between any two thresholds could be different for stars of different brightness. Since simple visual inspection of several plates seemed to indicate the presence of guiding problems (Sect. 2.3), we decided to check the in-plate magnitude equation and to correct for it in all plates measured with MAMA.

One reference threshold was selected for each plate, trying to maintain the widest dynamical range. The difference of photocenter positions between the reference threshold and any other was plotted as a function of measured flux. The differences were averaged in bins of flux, and a cubic spline drawn through these means was assumed to represent the relative magnitude equation from each threshold to the reference threshold.
In general, in-plate magnitude equations were small. In most cases, the effects remained below $\pm 1 \mu \mathrm{m}$, reaching $2 \mu \mathrm{m}$ at most in the bright end of some thresholds ( $\mathrm{T} 109$ ). In one case ( $\mathrm{T} 6573$ ), the equations reached $\pm 2 \mu \mathrm{m}$ or $\pm 3 \mu \mathrm{m}$ in some flux intervals. OCA plates (and, among them, that selected as master, OCA 3305) showed a virtually null in-plate magnitude equation.

The accepted positions of the stars for each plate were computed by transforming all the thresholds to the magnitude equation system of the reference one, and averaging the star position using the object area in each threshold as a weight. Of course, the correction of in-plate magnitude equation does not remove magnitude effects from the data: it simply reduces them to a common magnitude equation system (that of the reference threshold).

\subsection{Inter-plate magnitude equation}

The classical method for dealing with magnitude effects in plate-to-plate transformations, consists in including magnitude-dependent terms in the plate models, as in Eqs. (4) and (5). Terms $Q_{x}(x, y, m)$ and $Q_{y}(x, y, m)$ are usually built as non-complete polynomials, with several terms depending only on magnitude (linear and quadratic), and adding some mixed coordinate-magnitude dependent terms if optical aberrations such as coma are important. In some cases, colour-dependent terms have been used in the geometrical transformations.

As indicated by Kozhurina-Platais et al. (1995), this is not the best way of dealing with magnitude effects. Magnitude equations are not well represented by polynomial functions. Furthermore, there exists a real, intrinsic dependence of proper motions on magnitude: bright stars are statistically closer to the Sun and, so, display larger proper motions. This effect could be mistaken by the fit and introduced into the magnitude-dependent terms, eliminating physical information from the data and biasing the results. Last but not least, magnitude equations usually are stronger for bright stars, among which we expect the most important contribution of cluster members (Paper I). These members have a specific kinematical behaviour which should allow to distinguish them from the field population, and which could be misinterpreted and deformed by the classical approach that incorporates magnitude-dependent terms into the plate models.

For the reasons just explained, and following Kozhurina-Platais et al. (1995) and Dinescu et al. (1996), we dealt with inter-plate magnitude equations outside the ICOA. We evaluated and removed magnitude effects from all plates following several steps.

The first step implied the correction of modern plates (and the master plate among them) through comparison with contemporary CCD astrometric measurements. CCD star positions from Paper I are free of magnitude effects, due to the maximum-finding algorithm used to locate stars 
(FIND algorithm in DAOPHOT, Stetson 1987) and to the linearity of the detector. Being the CCD frames only a few arcmin across, other optical defects, such as coma, are not affecting the CCD astrometry. The comparison of star positions measured on individual CCD fields and on contemporary photographic plates allows us to evaluate and to correct for the magnitude-dependent shift due to magnitude equation. This process is described and applied to modern epoch plates in Sect. 6.2.1.

After that, old epoch plates were compared with the corrected modern epoch ones. Proper motions interfere with magnitude equation in this treatment of old epoch plates. CCD photometry from Paper I and the calculation of preliminary proper motions permitted us to disentangle real kinematical effects from magnitude equation in the treatment of old epoch plates, as described in Sect. 6.2.3.

\subsubsection{Modern epoch plates}

Since all modern epoch plates were obtained in a time interval of one year, any differences in star positions among them must be interpreted as caused not by proper motions, but by geometric effects or by magnitude equation. Assuming that the complete 2-D polynomials without photometric dependence for each source plate selected in Sect. 5.1 constitute a good representation of the geometric differences with the master plate, the residuals of the transformations should display only the magnitude equations.

The inspection of these residuals as a function of brightness displays the profile of the relative magnitude equations between each source plate and the master plate (Fig. 7 shows an example). These magnitude equations were represented by discrete points, calculated by averaging the residuals in flux intervals (incorporating a $3 \sigma$ clipping). The magnitude equation was represented by a cubic spline drawn through the points.

After determining and correcting magnitude equation for each modern epoch source plate, the residuals of the transformation to the master plate were checked as a function of colour and the quantities $x m$ and $y m$, where $m$ is a brightness estimator. No systematic trends were found.

At this point, all modern plates are affected by the same magnitude equation: that of the master plate. In order to remove this last effect, CCD astrometric data were used. The master plate positions can be directly compared with the CCD positions, but, having a complete set of four good quality plates in the same magnitude equation system, we preferred to average them before comparing with the $\mathrm{CCD}$, in order to reduce the random positional errors.

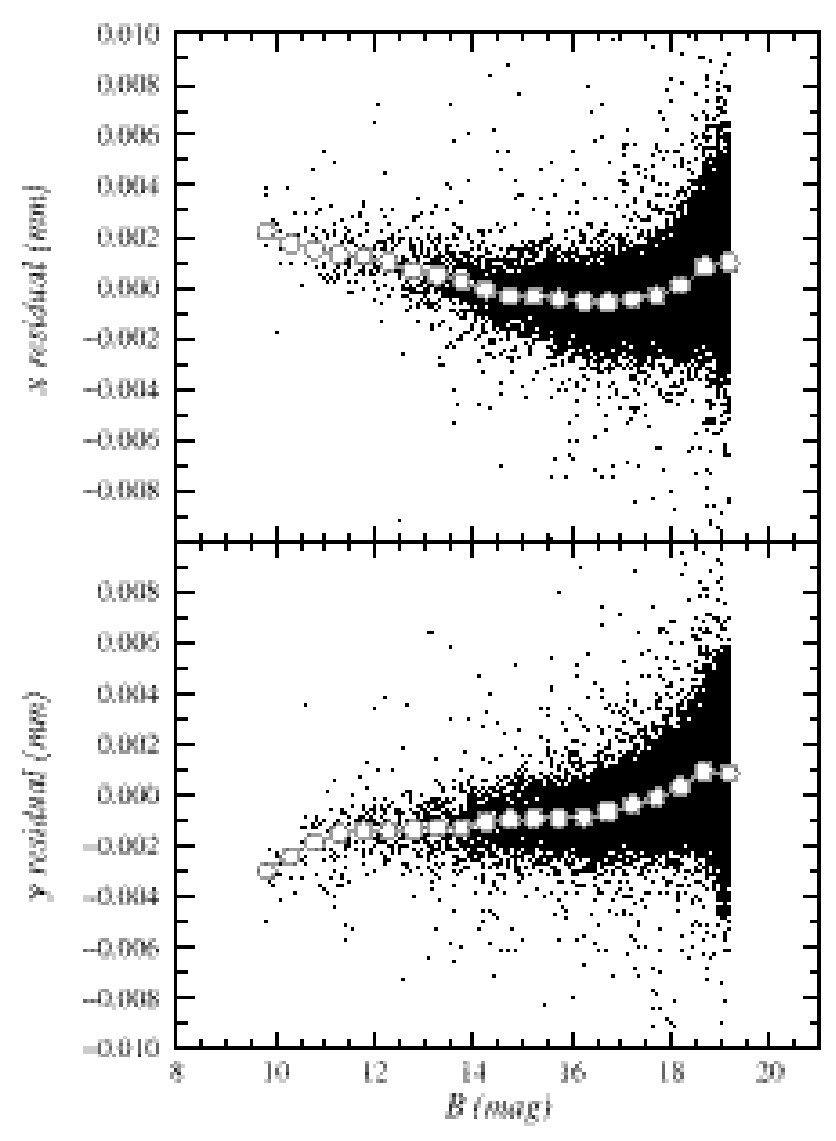

Fig. 7. The residuals of the transformation from source plate A 550 to the master plate OCA 3305 through a complete 4th degree polynomial without magnitude terms, as a function of flux. The graph permits the determination and removal of the relative magnitude equation among both plates

6.2.2. Master plate magnitude equation and CCD pseudo-plate

The comparison of the average modern plate with the CCD data was done independently for each CCD field. We took the star positions given by the FIND algorithm of DAOPHOT program (Stetson 1987). Most often, there were two different exposures of each field (long and short, as described in Paper I). In these cases, we adopted the star positions determined in the long exposure, except for saturated stars, whose positions were taken from the short exposures and transformed into the long exposure frames by means of a simple shift of coordinates. The shift was determined using all the stars in common among both frames.

Each CCD field was transformed onto the average modern plate using a complete 2-D polynomial without magnitude-dependent terms. The mean number of stars cross-identified between one CCD field and the average plate was 88 . Since the CCD fields slightly overlap, the comparison of the different transformed coordinates obtained for stars in the overlapped regions allowed us to 


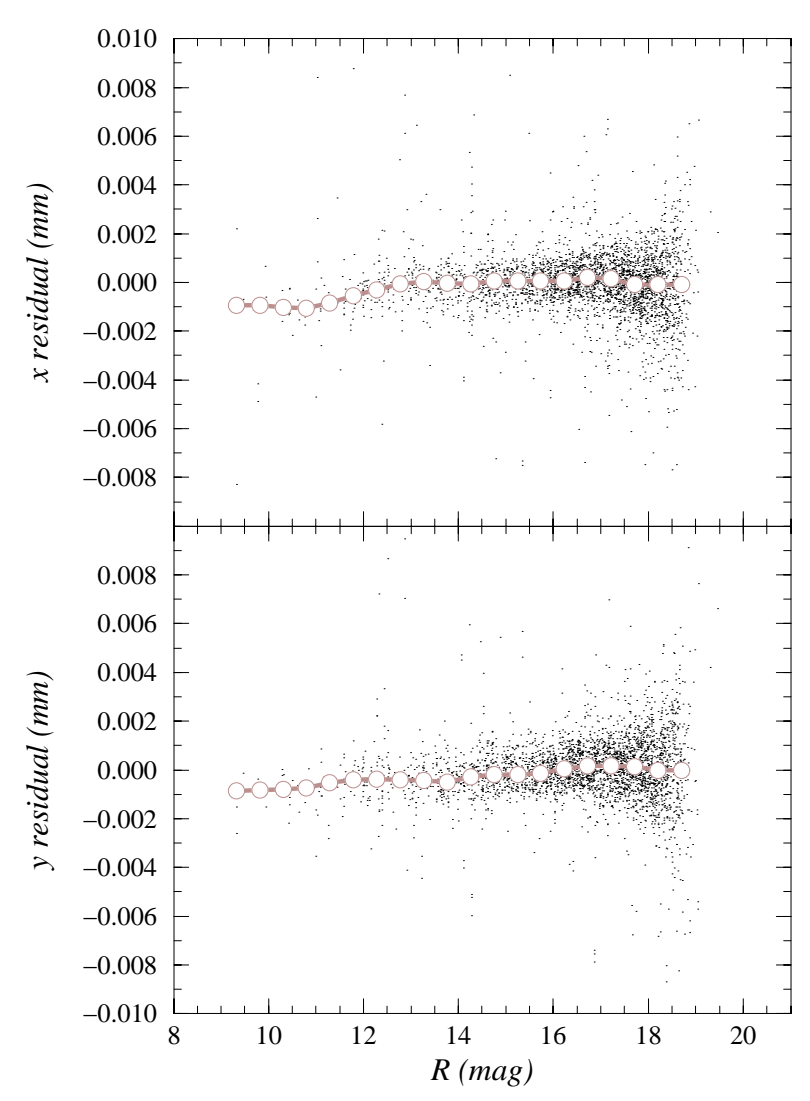

Fig. 8. Magnitude equation of the master plate, as deduced from comparison of the average modern photographic plate with CCD data

perform a realistic test of the absolute accuracy of the resulting star positions. A total of 735 stars were contained in more than one CCD field. The transformed positions found for 14 of them differed by more than $2 \mu \mathrm{m}\left(0.13^{\prime \prime}\right)$ in $x$, in $y$ or in both coordinates. For the other 721 stars, the differences of their positions displayed a standard deviation of $0.41 \mu \mathrm{m}\left(0.027^{\prime \prime}\right)$ in $x$ and in $y$.

The positions of stars matched in the overlapping areas were averaged, and the whole set of CCD astrometric information was assembled into a CCD pseudo-plate and introduced in the ICOA as one more plate.

Our CCD astrometry is free of magnitude effects. For this reason, the residuals of the transformations from the CCD frames onto the averaged modern photographic plate, when plotted as a function of magnitude, display the magnitude equation of the master plate (Fig. 8). This equation was determined using the same procedure described in Sect. 6.2.1. In this way, our modern epoch plates and master reference system are completely free of magnitude effects.

\subsubsection{Magnitude equation of old epoch plates}

Magnitude equation of old epoch plates is determined through comparison with the average modern epoch plate corrected for magnitude effects. For plates with a significant epoch difference with the master plate, proper motions not only introduce a higher dispersion in the plateto-plate transformations, but also interfere with the determination of magnitude equations, since there exists an intrinsic dependence of proper motion with magnitude. This drawback can be overturned by determining magnitude equations not using the whole set of cross-identified stars, but a subsample of stars known to have an homogeneous kinematical behaviour.

In order to select a kinematically homogeneous sample, we did our best to pick up a set of stars belonging to one of the star clusters present in the surveyed area. We selected as preliminary members those stars from Paper I close to the main sequence seen in the CCD colour-magnitude diagram. The resulting list was cleaned using additional kinematical data from raw proper motions.

The ICOA described in Sect. 4 was applied to derive these raw proper motions. The plate-to-plate transformations incorporated magnitude-dependent terms in quite a classical style. When solving Eqs. (6) and (7), we assigned a fixed weight to each plate, as just the focal length of each instrument (or, equivalently, the inverse of plate scales).

The stars previously selected as preliminary members from a photometric point of view, were analyzed regarding their raw proper motions. After suppressing a few high proper motion stars, the raw proper motions of the remaining objects were very similar in $x$, but two distinct groups in $y$, separated by about 5 mas $\mathrm{yr}^{-1}$, appeared. The spatial distribution of these two groups agreed rather well with what could be expected if the two clusters NGC 1750 and NGC 1758 were real. The stars with raw proper motion similar to that displayed by most of the stars in the area of NGC 1750, were selected to form the kinematically homogeneous sample of preliminary cluster members.

To begin the treatment of old epoch plates, they were grouped into sets of similar epoch and same telescope. Almost in all cases, these groups were formed by just one plate pair, except for AC plates, that were treated as only one set. A geometric transformation was computed to convert the plates in each group into one of them. The residuals of the transformations allowed us to determine and remove the relative magnitude equations inside each group. By averaging the plate sets, we reduced the uncertainty of star positions due to random measurement errors. Each averaged group of old plates was transformed into the average modern plate previously corrected for magnitude effects. The residuals of our kinematically homogeneous sample were analyzed in order to draw the magnitude equation of the old plate group. Figure 9 displays, as an example, the magnitude equation found for the average Palomar plate. 


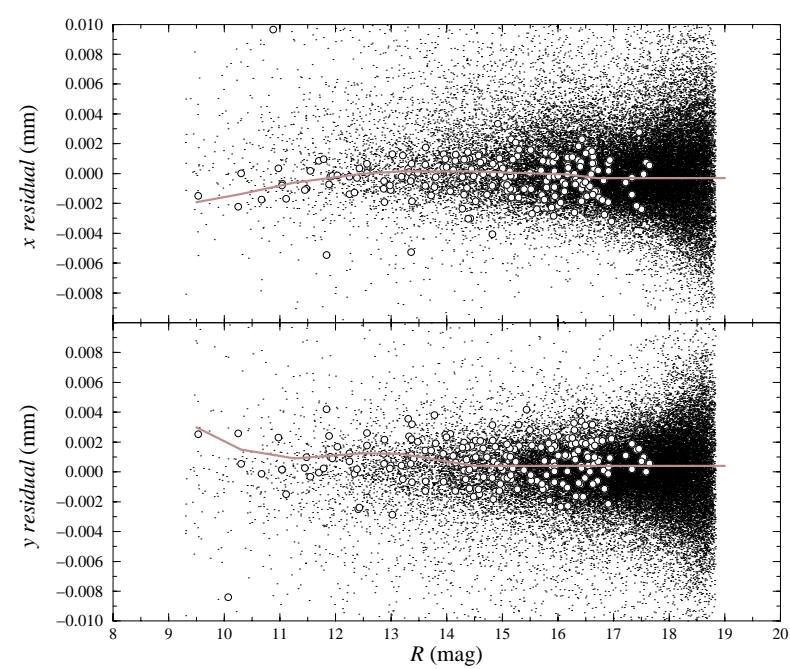

Fig. 9. Magnitude equation of the average POSS 1461 plate. The residuals of the transformation from POSS plate to the modern average plate are measured in the master plate system. Dots represent all the stars in common, while open circles correspond to the kinematically homogeneous sample used for defining the magnitude equation (solid line)

After correcting for magnitude effects, the residuals of the transformation from the old to the modern plates were also checked as a function of colour, $x m$ and $y m$. Only the residuals of Astrographic Catalog plates showed a very slight trend with coordinate $\times$ magnitude, which could be attributed to the presence of coma. The trend was small, and we decided not to correct for it.

\section{Final proper motions}

The data from the 27 individual photographic plates and the CCD pseudo-plate were introduced into the ICOA (Sect. 4) in order to determine relative proper motions. Since magnitude effects had been previously removed (Sect. 6), the plate-to-plate transformations included only the geometrical part of the plate models shown in Eqs. (4) and (5) $\left(Q_{x}=Q_{y}=0\right)$.

\subsection{Weighting system}

The heterogeneity of our astrometric material requires a careful weighting system, in order to combine all the data in the same process. We devised a somewhat sophisticated weighting system, to take into account the different plate scales, the possible different astrometric quality of plates obtained with the same telescope but with different emulsions (i.e., grain sizes) or in different seeing and guiding conditions, and to distinguish the intrinsically different accuracy of measurements of stars very different in brightness on the same plate.
As described in Sect. 6.2, for the determination and correction of inter-plate magnitude equations, plates were averaged in groups of equal epoch. Three modern Schmidt plates were transformed into the master plate magnitude equation system and averaged with it (Sect. 6.2.1). When computing the relative magnitude equation from each of these source plates to the master one, the dispersion around the magnitude equation was evaluated as a function of magnitude. The functions describing these dispersions, transformed to the master plate scale, were used for assigning magnitude-dependent weights to the stars in each modern epoch source plate. The master plate was individually compared to the CCD pseudo-plate, and the dispersion around this transformation was used for weighting purposes. For the CCD pseudo-plate, weights were assigned considering the dispersion of the positions in the zones overlapped among different CCD fields, in the master plate scale, as a function of magnitude.

Old epoch plates were averaged in groups before transforming them into the average modern plate for computing their magnitude equation (Sect. 6.2.3). The dispersion of the transformation inside each old epoch group, transformed to the master plate scale, was used for assigning magnitude-dependent weights to the star positions from these plates.

\subsection{Some details about the calculation}

Five iterations of the ICOA (Sect. 4) were enough for stabilizing the final proper motions. The transformationcrossing loop (Sect. 4.1) was, by far, the most timeconsuming step of the whole procedure. Only two internal iterations of the transformation-crossing loop (three at most, in some cases) were needed for all plates in all the ICOA iterations.

When performing the fits of Eqs. (6) and (7) for computing the proper motions, some star positions were rejected by the $3 \sigma$ clipping criterion. The different quality of the plates lead to clear tendencies in this rejection. Table 5 displays the statistics of detected and rejected points for each astrometric plate in this calculation step for the last ICOA iteration. As can be seen, the Heidelberg and several of the Astrographic Catalog plates are the less accurate from the astrometric point of view.

\section{The catalogue}

The final catalogue (Table 6, available only in electronic form) contains a total number of 51846 stars, all of them having positional information. 39762 stars have standard photographic photometry too (Sect. 3.1). A total number of 12029 stars do not have standard photometry, because they were not detected in the blue photometric plate, and no colour term could be applied. For these stars, the raw 
Table 5. Stars detected in each plate, and number of rejections in the calculation of proper motions in the last ICOA iteration

\begin{tabular}{llll}
\hline Plate & Detections & Rejections & \% rejected \\
\hline A 550 & 29022 & 131 & 0.45 \\
A 573 & 31187 & 194 & 0.62 \\
\hline OCA 3305 & 52865 & 14 & 0.03 \\
OCA 3308 & 18741 & 320 & 1.71 \\
\hline POSS 1461-O & 33746 & 1031 & 3.06 \\
POSS 1461-E & 41533 & 1104 & 2.66 \\
\hline T 109 & 10189 & 6 & 0.06 \\
T 111 & 5097 & 6 & 0.12 \\
T 6573 & 6969 & 4 & 0.06 \\
T 6574 & 4563 & 1 & 0.02 \\
\hline B 1118 & 1433 & 580 & 40.48 \\
B 4191 & 891 & 395 & 44.33 \\
\hline AC 2238 & 133 & 16 & 12.03 \\
AC 2239 & 65 & 5 & 7.69 \\
AC 2338 & 215 & 23 & 10.70 \\
AC 2438 & 341 & 77 & 22.58 \\
AC 2439 & 62 & 4 & 6.45 \\
\hline S 470 & 532 & 0 & 0.00 \\
S 471 & 531 & 0 & 0.00 \\
S 526 & 509 & 38 & 7.47 \\
S 527 & 507 & 40 & 7.89 \\
S 60001 & 439 & 18 & 4.10 \\
S 60002 & 461 & 39 & 8.46 \\
S 83001 & 519 & 3 & 0.58 \\
S 83002 & 511 & 9 & 1.76 \\
S 86006 & 538 & 0 & 0.00 \\
S 86007 & 537 & 1 & 0.19 \\
\hline CCD pseudo-plate & 1486 & 0 & 0.00 \\
\hline & & &
\end{tabular}

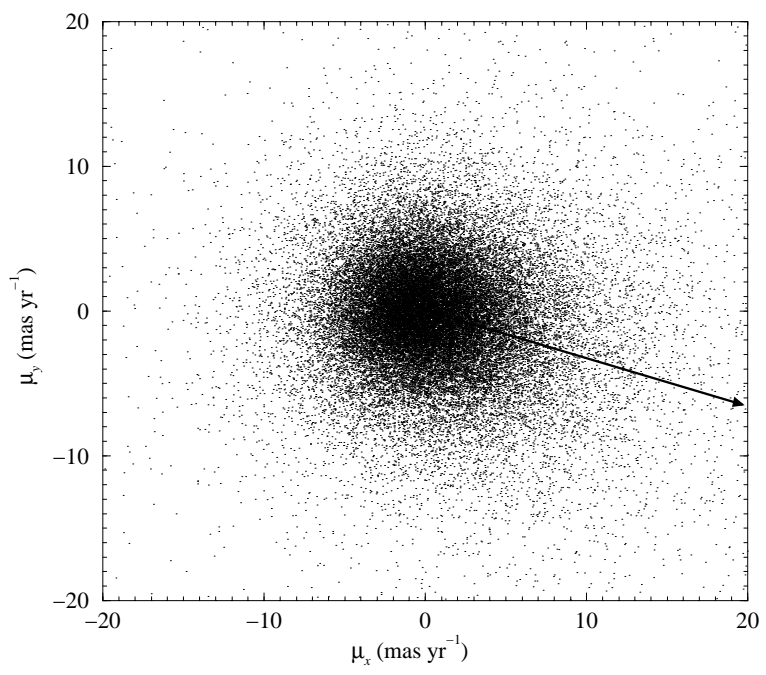

Fig. 10. Vector point diagram for the whole set of relative proper motions in the master plate reference system. The arrow signs the direction of the antiapex of the solar motion. The axes alignment is $x \leftrightarrow-\delta, y \leftrightarrow-\alpha$

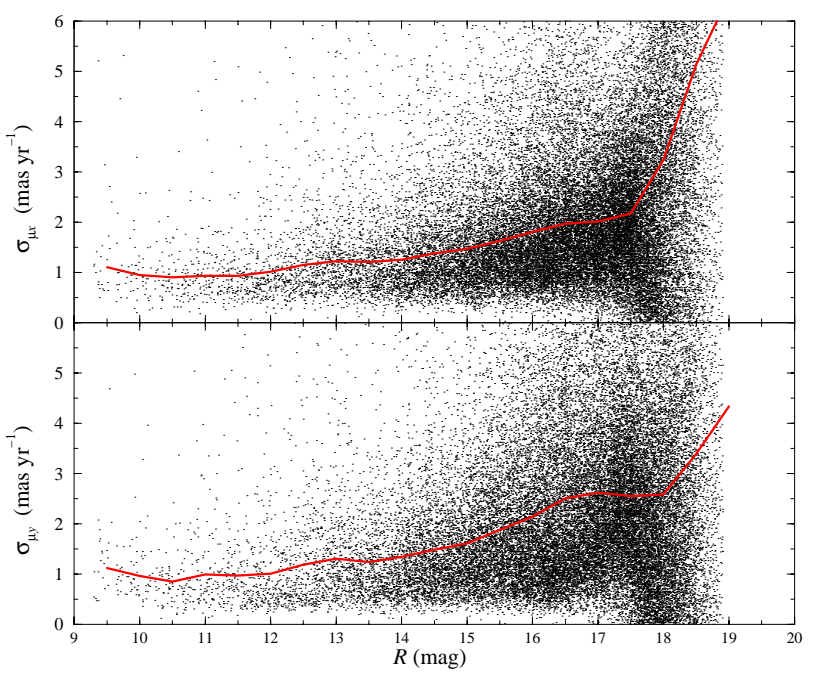

Fig. 11. Internal error of the proper motions as a function of $R$ photographic magnitude. The line traces the mean error computed in half magnitude bins, applying a $3 \sigma$ clipping. The axes alignment is $x \leftrightarrow-\delta, y \leftrightarrow-\alpha$

$R$ magnitude (Sect. 3.3) computed from the master plate was included in the catalogue as brightness indicator. 55 stars do not have standard nor raw photometry, for being too bright: they are labelled with $R=0$ in the catalogue. Proper motions were computed for 45036 stars, 37129 of them using three or more different plates (31257 stars having complete standard photographic photometry). As said in Sect. 4.2, proper motions were computed provided plates span a minimum of 30 years. The catalogue contains, for each star, the following data: an identification number (that assigned in MAMA original files to the objects in the master plate), the coordinates in the master plate reference system, the proper motions with their standard errors, and the $B V R$ photographic photometry. Cross-identifications with the most widespread star catalogues and with previous studies of the zone (Cuffey 1937; Straižys et al. 1992, Paper I) are given in notes to Table 6. Figure 10 represents the vector point diagram (VPD) of the whole set of relative proper motions. The elongation in the VPD due to the solar motion is evident.

\subsection{Internal astrometric errors}

The least squares fits described in Sect. 4.2 provide a standard error on the coefficients when they are computed using more than two plates. These errors show a strong dependence with apparent magnitude. Figure 11 displays this behaviour, and Table 7 shows the mean error in $R$ magnitude intervals, computed applying a $3 \sigma$ clipping.

As could be expected, the internal errors are strongly dependent on the number of plates used for the calculation. Table 8 displays the average error of proper motions as a function of the number of plates. There is no clear 
Table 7. Internal error of the proper motions $\sigma_{\mu x}$ and $\sigma_{\mu y}$ (mas $\mathrm{yr}^{-1}$ ) averaged in $R$ magnitude intervals, applying a $3 \sigma$ clipping. The axes alignment is $x \leftrightarrow-\delta, y \leftrightarrow-\alpha$

\begin{tabular}{lll}
\hline$R$ range & $\sigma_{\mu x}$ & $\sigma_{\mu y}$ \\
\hline$<10$ & 1.10 & 1.13 \\
$10-11$ & 0.90 & 0.85 \\
$11-12$ & 0.93 & 0.97 \\
$12-13$ & 1.15 & 1.19 \\
$13-14$ & 1.21 & 1.24 \\
$14-15$ & 1.38 & 1.48 \\
$15-16$ & 1.63 & 1.88 \\
$16-17$ & 1.97 & 2.51 \\
$17-18$ & 2.17 & 2.55 \\
$18-19$ & 5.14 & 4.34 \\
\hline
\end{tabular}

Table 8. Average internal error of the proper motions $\sigma_{\mu x}$ and

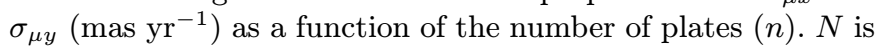
the number of stars. The axes alignment is $x \leftrightarrow-\delta, y \leftrightarrow-\alpha$

\begin{tabular}{llll}
\hline$n$ & $\sigma_{\mu x}$ & $\sigma_{\mu y}$ & $N$ \\
\hline 3 & 4.49 & 3.42 & 10588 \\
4 & 2.58 & 3.10 & 3431 \\
5 & 2.03 & 2.69 & 6493 \\
6 & 2.10 & 2.92 & 7090 \\
7 & 2.04 & 2.28 & 3087 \\
8 & 2.08 & 2.25 & 2517 \\
9 & 1.97 & 1.85 & 1952 \\
10 & 1.84 & 1.61 & 1309 \\
11 & 1.70 & 1.47 & 135 \\
12 & 2.61 & 1.32 & 11 \\
13 & 2.30 & 1.69 & 14 \\
14 & 1.08 & 0.96 & 19 \\
15 & 1.33 & 1.10 & 26 \\
16 & 1.18 & 0.94 & 44 \\
17 & 1.09 & 0.70 & 86 \\
18 & 0.89 & 0.81 & 93 \\
19 & 0.88 & 0.78 & 110 \\
20 & 0.88 & 0.85 & 73 \\
21 & 1.27 & 1.13 & 44 \\
22 & 0.83 & 0.88 & 15 \\
\hline
\end{tabular}

tendency of the errors as a function of the distance to the master plate center, nor for the whole sample, nor for the brightest stars $(R<16 \mathrm{mag})$. Figure 12 displays the histogram of the error distribution for the whole sample, and for the stars brighter than $R=16$ mag.

The mode of the proper motion errors is below 1 mas $\mathrm{yr}^{-1}$. The achieved precision is good, considering the diversity of our plate material and the short focal length of several telescopes used; the careful treatment allowed the removal of systematic effects.

\subsection{Transformation into the equatorial system}

The astrometric catalogue was transformed into FK5 and ICRS reference systems through comparison with the following catalogues: Positions and Proper Motions

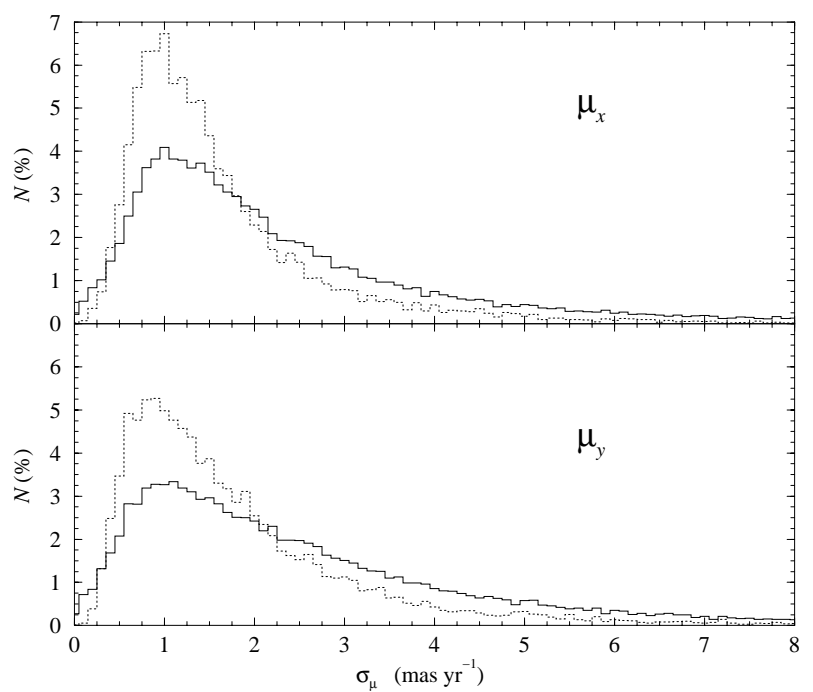

Fig. 12. Histograms of the proper motion error distribution for the whole sample (solid lines) and for the stars brighter than

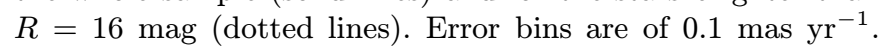
Number of stars in each bin is expressed as percentage of the total number of stars. The axes alignment is $x \leftrightarrow-\delta, y \leftrightarrow-\alpha$

Table 9. Transformation to the equatorial system: number of reference stars $(N)$ and standard deviation of the residuals of the transformations $\left(\sigma_{\alpha}\right.$ and $\left.\sigma_{\delta}\right)$ for the reference catalogues PPM, HIP and TYC

\begin{tabular}{llll}
\hline Catalogue & $N$ & $\sigma_{\alpha}\left({ }^{\prime \prime}\right)$ & $\sigma_{\delta}\left({ }^{\prime \prime}\right)$ \\
\hline PPM & 61 & 0.203 & 0.241 \\
HIP & 19 & 0.148 & 0.111 \\
TYC & 160 & 0.138 & 0.106 \\
\hline
\end{tabular}

Catalogue (PPM; Röser \& Bastian 1989), Tycho and Hipparcos (TYC and HIP; ESA 1997). Equatorial coordinates from these catalogues were transformed to the master plate epoch by taking into account the proper motions and, after that, were converted to standard coordinates through the usual gnomonic projection (see, for instance, Van de Kamp 1967). The resulting virtual plates were cross-identified with our astrometric catalogue, and transformation equations were computed by means of a procedure similar to the transformation-crossing loop described in Sect. 4.1. The models applied were third degree (PPM and TYC) or second degree (HIP) complete 2-D polynomials without magnitude-dependent terms. The resulting coefficients evidenced a very good alignment of our $x$ coordinate with $-\delta$, and $y$ with $-\alpha$. Table 9 shows the number of stars in common with the catalogues, and the standard deviation of the residuals of the fits.

The transformation coefficients obtained allowed us to compute equatorial coordinates for the master plate epoch and J2000.0 equinox, referred to FK5 and to ICRS, for all stars in our astrometric catalogue. After that, the $(x, y)$ coordinates from our catalogue were modified by adding 


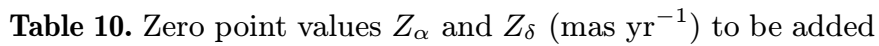
to our proper motions, to convert them to FK5 and ICRS, after aligning them with the coordinate axes of these reference systems. $N$ is the number of stars used to compute the parameters

\begin{tabular}{llll}
\hline Catalogue & $Z_{\alpha}$ & $Z_{\delta}$ & $N$ \\
\hline FK5 (PPM) & $-4.2 \pm 0.6$ & $-6.8 \pm 0.7$ & 46 \\
ICRS (HIP) & $-0.5 \pm 1.0$ & $-7.5 \pm 0.7$ & 13 \\
ICRS (TYC) & $+0.6 \pm 1.3$ & $-6.9 \pm 1.4$ & 48 \\
\hline
\end{tabular}

their centennial proper motions, and the resulting positions $\left(x+\mu_{x}, y+\mu_{y}\right)$ were converted again into the equatorial system by means of the same transformation coefficients. The comparison of the equatorial positions in the master plate epoch and in the master plate epoch $+100 \mathrm{yr}$ allowed an easy conversion of our $\left(\mu_{x}, \mu_{y}\right)$ proper motions into $\left(\mu_{\alpha}, \mu_{\delta}\right)$ in the FK5 and ICRS reference systems, except for a zero point shift. The zero point shifts (Table 10) were determined comparing the just obtained $\left(\mu_{\alpha}, \mu_{\delta}\right)$ with the proper motions quoted in the reference catalogues. The calculation of the zero point shifts to ICRS is affected by lack of a good number of reference stars in the case of HIP, and by lack of accuracy of proper motions in the case of TYC.

Table 11 (available only in electronic form) contains, for each star in our catalogue: the identification number, the astrometric position and the proper motions in the ICRS and FK5 systems. For the transformation of proper motions to ICRS, the zero points computed from comparison with HIP catalogue were applied.

\section{Astrometric and photometric signature of the clusters}

We performed a simple analysis of the final photometric and astrometric catalogue, applying strictly spatial criteria for the separation of different subsamples, in a similar way as was done in Paper I for the analysis of CCD photometry. We adopted the cluster positions and diameters used in Paper I: a diameter of $28^{\prime}$ centered at $\alpha_{2000}=5^{\mathrm{h}} 4.0^{\mathrm{m}}, \delta_{2000}=+23^{\circ} 41^{\prime}$ for NGC 1750; and a diameter of $8^{\prime}$ centered at $\alpha_{2000}=5^{\mathrm{h}} 4.7^{\mathrm{m}}, \delta_{2000}=+23^{\circ} 48^{\prime}$ for NGC 1758. Stars in the zone where the two circles overlap, were assigned to NGC 1758. Figure 13 shows the proper motion histogram and the colour-magnitude diagram for the stars assigned to NGC 1758, to NGC 1750 and to the remaining zone (assumed to represent the field), using only the stars with $R<16$ mag and with proper

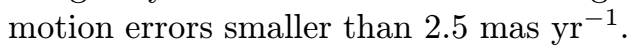

Although this raw spatial classification implies contamination due to field stars in the clusters zones as well as to the strong overlap of the two possible clusters, the histograms and colour-magnitude diagrams allow to draw some very interesting conclusions.
The field population shows a disperse colourmagnitude diagram, without any well defined main sequence. The field proper motion distributions, in $x$ as well as in $y$, are smooth in shape, clearly skewed as expected from the inspection of Fig. 10. The colour-magnitude diagrams corresponding to the cluster areas show clearly defined main sequences, with characteristics in agreement with the conclusions pointed out in Paper I: this spatial classification of photographic photometric data does not allow us to distinguish clear differences among the sequences of NGC 1750 and NGC 1758, except for the known fact that the second cluster lacks bright stars.

The kinematical information adds new insights to the photometric data. An overdensity of stars is apparent in the proper motion histograms for the $x$ coordinate, when comparing the clusters areas with the field population. This overdensity is found close to zero proper motion, but shows a slightly positive trend in the NGC 1758 area, and a negative tendency in NGC 1750. This feature of the $\mu_{x}$ histograms could be attributed to the presence of one or possibly two clusters in the zones. The $\mu_{y}$ histograms clarify the situation. The stellar overdensity has different behaviour in the two zones, being the maximum overdensities in NGC 1750 and NGC 1758 separated by

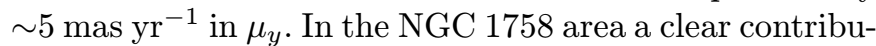
tion of stars sharing NGC 1750's proper motion indicates the contamination due to the overlap.

The presence of two kinematically different groups, with spatial distributions coincident with those assigned to the clusters from visual (spatial stellar density) as well as from photometric criteria, moves us to assert the reality of two separated open clusters in the zone. These clusters can be identified without ambiguity with the objects NGC 1750 and NGC 1758 of Dreyer's (1888) catalogue. No astrometric nor photometric indication of the existence of NGC 1746 was found. This signature of the objects moves us to perform a deeper analysis of the astrometric and photometric data presented in this article and in Paper I, with the aim of assigning individual membership probabilities to the stars and determining the fundamental physical parameters of the two clusters. The strong spatial overlap of the two clusters and their sparseness requires a careful segregation procedure. The third and last article of this series will be devoted to this goal.

\section{Conclusions}

A photographic astrometric and photometric catalogue was constructed for a total number of 51846 stars in an area of $2.3^{\circ} \times 2.3^{\circ}$ around the positions of NGC 1750 and NGC 1758. CCD stellar positions from different CCD frames were assembled into a pseudo-plate through a method that ensured astrometric uniformity. This allowed us to use the whole set of CCD positions as one more plate for astrometric purposes. The interaction of CCD and photographic information, combined with a careful treatment 

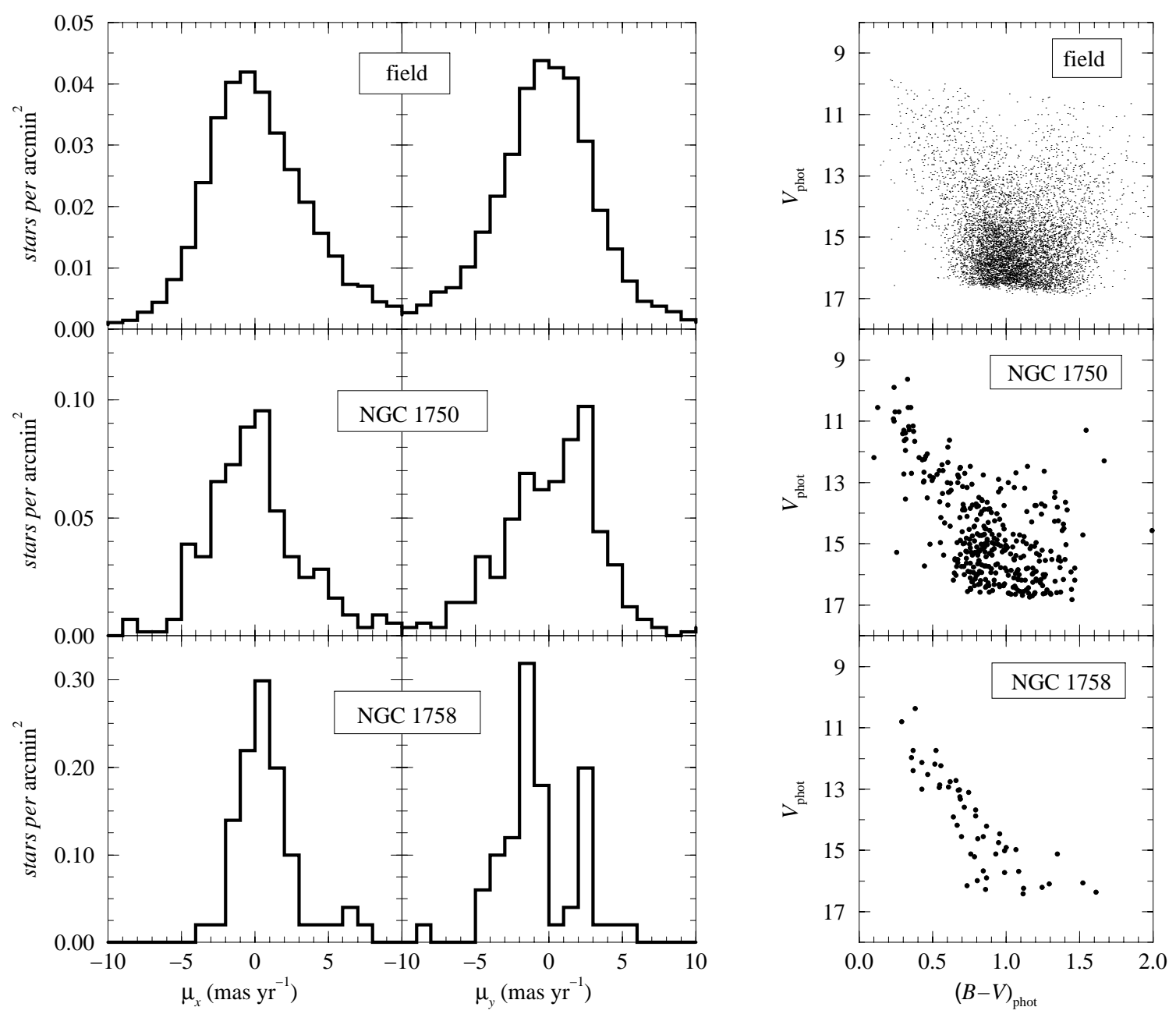

Fig. 13. Proper motion histograms and photographic colour-magnitude diagrams for the stars assumed to belong to the field (top), NGC 1750 (center) and NGC 1758 (bottom). The sample has been limited to $R<16$ mag and to stars with proper

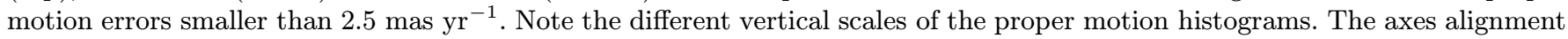
is $x \leftrightarrow-\delta, y \leftrightarrow-\alpha$

of the data, allowed the complete removal of magnitude effects from the astrometric positions and proper motions. A new implementation of the iterative central overlap algorithm was devised for this study.

One film was included among our plate material. The tests performed indicated that this detector has a good photometric quality, but it is not useful for astrometry. This casts doubt on the reliability of film-based detectors for wide-field astrometry, although further tests on other film plates are needed in order to clarify this point.

In spite of the inhomogeneity of the plate material, a detailed consideration of the plate-to-plate transformation models allowed us to achieve a good proper motion precision. The resulting relative proper motions have a typical standard error of $\sim 1$ mas $\mathrm{yr}^{-1}$ for well-measured stars in the magnitude interval $R=9-14$ mag.
Positions and proper motions were transformed into the FK5 and ICRS reference systems through comparison with PPM, Tycho and Hipparcos catalogues.

Photographic Johnson-Cousins $B V R$ photometry was obtained for 39762 stars by calibrating four filtered modern epoch Schmidt plates, using CCD photometry as reference. The resulting photometry has a mean precision of $0.08 \mathrm{mag}$ in $B$ and $V$, and $0.04 \mathrm{mag}$ in $R$. The catalogue limiting magnitudes are $B \approx 19.5, V \approx 18.5$ and $R \approx 18$ mag.

The analysis of the data from a spatial point of view allowed us to distinguish without ambiguity two real open clusters in the field. The objects are sparsely populated and show a strong spatial overlap. For their spatial positions and distributions, and for their apparent main sequences, the clusters can be identified with NGC 1750 and NGC 1758. The entry number 1746 of Dreyer's 
NGC catalogue most probably does not correspond to any real object in this field.

Further analysis of the data presented in this paper will allow to assign membership probabilities and to determine the main astrophysical parameters of both clusters.

Acknowledgements. We express our thanks to W. Van Altena and to all members of the Astrometry Group of the Department of Astronomy at Yale University for their help for the treatment of the data. Special thanks to I. Platais and T. Girard for their sharp comments and advises for dealing with magnitude equations. The authors are very thankful to C. Alard for providing us with his original C codes, to N. Robichon for his comments and suggestions, and to R. Chesnel for his patience and efficiency at plate scannings. We are also very grateful to E. Schilbach and S. Röser for their loan of Tautenburg Schmidt and Bruce Astrograph plates for this work. Thanks also to S.E. Urban for providing us with the original individual measurements of Astrographic Catalog plates from Paris zone. Thanks a lot to K. Birkle, J. Zamorano and Ó. Alonso, who kindly exposed and developed for us the CAHA Schmidt plates for this work. D.G.-E. is indebted to the staffs of the Centre d'Analyse des Images and the Astronomy Department of Yale University for their hospitality and unvaluable help, without which this work would not have been possible. This work has been supported by CYCIT under contract ESP95-0180 and DGPC. D.G.-E. acknowledges partial support by the Spanish Ministerio de Educación y Ciencia (Ref. AP92 30526274) and by the Comissió Interdepartamental per a la Recerca i la Tecnologia (Generalitat de Catalunya, Ref. 1996BEAI200346).

\section{References}

Alard C., Guibert J., 1997, A\&A 326, 1

Berger J., Cordoni J.P., Fringant A.M., et al., 1991, A\&AS 87, 389

Cudworth K., Martin S., DeGioia-Eastwood K., 1993, AJ 105, 1822

Cuffey J., 1937, Annals Harvard Obs. 105, 403

Dinescu D., Girard T., Van Altena W., Yang T., Lee Y., 1996, AJ 111, 1205
Dreyer J.L.E., 1888, New General Catalogue of Nebulae and Clusters of Stars, in Mem. R. Astron. Soc., 49

Eichhorn H., 1974, Astronomy of Stars Positions. Frederick Ungar Publishing Co.

Eichhorn H., 1988, ApJ 334, 465

Eichhorn H., Jefferys W.H., 1971, Pub. Leander McCormick Obs. 16, 267

ESA, 1997, The Hipparcos and Tycho Catalogues, ESA-SP 1200

Galadí-Enríquez D., Jordi C., Trullols E., Ribas I., 1998, A\&A 333, 471 (Paper I)

Guibert J., Soubiran C., Geffert M., 1990, in: Colloque André Danjon, Capitaine N., Débarbat S. (eds.). Paris, p. 211

Hunstead R.W., 1973, "Accurate optical positions using the Palomar sky atlas", in IAU Symp. 61, Perth

Li Hen, 1954, Annales de l'Observatoire Astronomique de ZǒSè XXIII, p. 51

Röser S., Bastian U., 1989, PPM-Positions and Proper Motions-North, Astron. Rechen. Inst. Heidelberg

Jones B.F., Walker M.F., 1988, AJ 95, 1755

Kozhurina-Platais V., Girard T., Platais I., et al., 1995, AJ 109,672

Schilbach E., Robichon N., Souchay J., Guibert J., 1995, A\&A 299,696

Soubiran C., 1992, A\&A 259, 394

Stetson P.B., 1987, PASP 99, 191

Straižys V., Černis K., Meištas E., 1992, Baltic Astron. 1, 125

Tian K.P., Zhao J.L., Van Leeuwen F., 1994, A\&AS 105, 15

Tucholke H.-J., 1992, A\&AS 93, 293

Tucholke H.-J., Scholz R.-D., Brosche P., 1994, A\&AS 104, 161

Urban S.E., 1997, Proceedings of IAU Colloquium 165, Dynamics and Astrometry of Natural and Artificial Celestial Bodies. Kluwer Academic Publishers, p. 493

Urban S.E., Corbin T.E., 1996, A\&A 305, 989

Urban S.E., Corbin T.E., Wycoff G.L., 1997, AC 2000: ACT Reference Catalogue, United States Naval Observatory

Van Altena W., Lee J.T., Lee J.-F., Lu P.K., Upgren A.R., 1988, AJ 95, 1744

Van de Kamp P., 1967, in Principles of Astronomy, Chap. 5 W.H. Freeman and Company

Zhao J.L, He Y.P., 1990, A\&A 237, 54 